\title{
The effect of grain orientation on fretting fatigue plasticity and life prediction
}

\author{
O.J. McCarthy ${ }^{\mathrm{a}}$, J.P. McGarry ${ }^{\mathrm{b}}$, S.B. Leen ${ }^{\mathrm{a}, *}$ \\ a Mechanical Engineering, NUI Galway, Ireland \\ ${ }^{\mathrm{b}}$ Biomedical Engineering, NUI Galway, Ireland
}

\section{A R T I C L E I N F O}

\section{Article history:}

Received 28 June 2013

Received in revised form

30 August 2013

Accepted 30 September 2013

Keywords:

Fretting fatigue life

Microstructure sensitive

316L stainless steel

Cyclic plasticity

\begin{abstract}
A B S T R A C T
A study on crystal and $J_{2}$ plasticity prediction of fretting fatigue is presented, using a microstructuresensitive fatigue parameter for crystal plasticity crack nucleation and a critical-plane (multiaxial) fatigue parameter for $J_{2}$ plasticity. A short crack propagation methodology is also implemented. The effect of grain orientation on nucleation life is shown to be significant for fretting fatigue. $J_{2}$ plasticity generally predicts conservative lives. Crystal plasticity is superior in terms of (i) accuracy of life prediction, (ii) ability to facilitate wear prediction and (iii) capturing the key effects of substrate fatigue stress and grain orientation on life. The crystal plasticity model facilitates new insight into interaction between grain orientation, fatigue stress amplitude and fretting surface damage vis-à-vis fretting fatigue life.
\end{abstract}

(c) 2013 Elsevier Ltd. All rights reserved.

\section{Introduction}

Contact fatigue and, more specifically, fretting fatigue (FF) are common problems in engineering contacts, particularly highly-loaded contacts, across a wide range of industrial and other engineering applications. Obvious examples include aeroengine dovetail joints and spline couplings [1], biomedical implants [2], shaft-hub connections [3] and fastener connections [4]. A key ongoing challenge in the design against FF is the development of reliable predictive methods for crack nucleation. Fretting cracks have been identified at length-scales competitive with the material micro-structure, suggesting the need for a micro-mechanical approach. Length-scales have been identified as a key aspect in the development of reliable life prediction methods for FF, to capture stress gradient effects associated with the contact size effect [5], for example. Araujo and Nowell [5] identified the need for volumeaveraging of critical-plane fatigue indicator parameters (FIPs), in the context of classical elasticity (analytical) solutions for fretting stress distributions, to capture the contact size effect.

\footnotetext{
Abbreviations: FF, fretting fatigue; FIP, fatigue indicator parameter; FE, finite element; COF, coefficient of friction; CP, crystal plasticity; SS, stainless steel; NLKH, non linear kinematic hardening; UMAT, user material subroutine; $\mathrm{FCI}$, fatigue crack initiation; SEM, scanning electron microscope; ESBD, electron back scatter diffraction; SWT, Smith Watson Topper; PF, plain fatigue; SCG, short crack growth; SIF, stress intensity factor; FFRF, fretting fatigue reduction factor; Stdev, standard deviation; CPFE, crystal plasticity finite element

* Corresponding author.

E-mail address: Sean.leen@nuigalway.ie (S.B. Leen).
}

The averaging dimension was shown to be broadly associated with the key micro-structural dimension of grain size. Sum et al. [6] subsequently demonstrated that mesh refinement techniques within a finite element (FE) based critical-plane FIP approach (Smith-Watson-Topper and Fatemi-Socie) could achieve the same result, i.e. capture the stress gradient and hence contact size effect. In other words, it was demonstrated that the FE mesh refinement process was equivalent to an averaging approach.

Fretting can typically be categorised into three different sliding regimes, namely, partial, mixed and gross slip, primarily dependent on normal load $(P)$, displacement amplitude and coefficient of friction (COF) [7]. Fig. 1(a) shows the relationship between normal load and displacement amplitude for the different slip regimes. Fig. 1(b) illustrates the material damage associated with each respective fretting regime. A similar fretting map has been presented by Vingsbo and Soderberg [8] (Fig. 2) where the effect of fretting regime is plotted in terms of number of cycles to failure and wear rate. Experimental data has been presented by Jin and Mall [9], for example, to corroborate the key effect of increasing fatigue life with increasing slip amplitude on transition from partial to gross slip. Madge et al. [10] have demonstrated that this effect can be predicted using a wear-fatigue approach. This work involved the explicit simulation of wear-induced material removal and simultaneous computation of fatigue damage via Miner's rule due to the wear-induced evolution of contact stress and strain distributions. Madge et al. [10] demonstrated the importance of contact stress re-distribution, and associated fatigue damage re-distribution, due to widening of the contact region vis-à-vis the competition between material removal and crack propagation. 


\begin{tabular}{|c|c|c|c|}
\hline \multicolumn{2}{|c|}{ Nomenclature } & \multirow{3}{*}{$\begin{array}{l}\sigma_{\max } \\
\sigma_{f^{\prime}} \\
b \\
\Delta \varepsilon_{p}\end{array}$} & \multirow{2}{*}{$\begin{array}{l}\text { maximum stress } \\
\text { fatigue strength coefficient } \\
\text { fatigue strength exponent }\end{array}$} \\
\hline$P$ & normal load & & \\
\hline$d$ & grain size & & plastic strain range \\
\hline$\delta$ & fretting stroke & $c$ & fatigue ductility exponent \\
\hline$a_{0}$ & initial contact semi-width & $\Delta \varepsilon$ & strain range \\
\hline$f$ & von Mises yield function & $a_{t h}$ & threshold crack length \\
\hline$d p$ & increment in effective plastic strain & $\Delta K_{t h}$ & threshold stress intensity factor \\
\hline$\sigma_{e}$ & von mises equivalent stress & $\sigma_{f l}$ & stress fatigue limit \\
\hline$\sigma^{\prime}$ & deviatoric stress tensor & $a$ & crack length \\
\hline $\boldsymbol{x}$ & backstress tensor & $N_{P}$ & number cycles to propagate \\
\hline C & initial hardening modulus & $\Delta K$ & stress intensity factor \\
\hline$\gamma$ & modulus rate of decay & $d a / d N$ & change in crack length per cycle \\
\hline$k$ & initial cyclic yield stress & $\Delta K_{e f f}$ & effective stress intensity factor \\
\hline$\alpha$ & slip system & $\Delta K_{\mathrm{I}}$ & stress intensity factor in mode I \\
\hline$\beta$ & slip system not equal to $\alpha$ & $\Delta K_{\mathrm{II}}$ & stress intensity factor in mode II \\
\hline$\dot{\gamma}^{\alpha}$ & shear strain rate on slip system $\alpha$ & $C, m$ & Paris crack growth constants \\
\hline$\dot{a}$ & reference strain rate & $\sigma_{\min }$ & minimum stress \\
\hline$\tau^{\alpha}$ & resolved shear stress on slip system $\alpha$ & $\Delta N$ & cycle jumping factor \\
\hline$g^{\alpha}$ & strain hardness on slip system $\alpha$ & $D_{\text {fret2 }}$ & fretting fatigue damage parameter \\
\hline$m$ & rate sensitivity exponent & $\tau \delta$ & frictional work value \\
\hline$h_{\alpha \beta}$ & strain hardening modulus & $\tau \delta_{t h}$ & frictional work threshold value \\
\hline$g_{0}$ & critical resolved shear stress & $C, n$ & $d_{\text {fret2 }}$ constants \\
\hline$g_{\infty}$ & saturation stress & $N_{i}^{e x p}$ & number of cycles to crack initiation \\
\hline$h_{0}$ & initial hardening modulus & $N_{S C G}$ & number of short crack growth cycles \\
\hline$\gamma_{a}$ & accumulated shear strain & $a_{c}$ & critical fracture length \\
\hline$p$ & accumulated plastic slip & $K_{I c}$ & fracture toughness \\
\hline $\mathbf{L}^{p}$ & plastic velocity gradient & $k$ & wear coefficient \\
\hline$\dot{p}$ & effective plastic slip rate & $V$ & wear volume \\
\hline $\mathbf{S}^{\alpha}$ & slip direction vector & $S$ & total sliding distance \\
\hline $\mathbf{n}^{\alpha}$ & slip normal vector & $\Delta \sigma_{x x}$ & stress amplitude parallel to the loading direction \\
\hline$p_{\text {crit }}$ & critical accumulated plastic slip & $\Delta \sigma_{x y}$ & stress amplitude perpendicular to the loading direction \\
\hline$N_{i}$ & number of cycles to crack initiation & $Y$ & Geometry factor for SIF \\
\hline$p_{c y c}$ & accumulated plastic slip per cycle & & \\
\hline
\end{tabular}

More recent work by Ding et al. [11], on experimental and computational modelling of wear and fatigue crack nucleation for Ti-6Al-4V, following similar fretting-plasticity work by Ambrico and Begley [12], for example, has demonstrated the need for cyclic plasticity modelling in the prediction of fretting crack nucleation, due to the induction of plasticity by wear. This work, however, among others, highlighted the short length-scales of identified cracks, competitive with the grain morphology, thus suggesting the need for micro-structural plasticity modelling as a more scale-consistent approach to the prediction of contact variable distributions and crack nucleation.

A number of authors have indeed applied crystal plasticity (CP) constitutive models to FF. McDowell and co-workers [13-15], for example, have used $\mathrm{CP}$ to develop plastic strain maps that qualitatively agree with experimentally-observed crack locations and orientations for fretting wear and fatigue testing of Ti-6Al-4V. Cailletaud and colleagues have also modelled the cyclic plasticity behaviour of Ti-6Al-4V under fretting wear conditions using a polycrystal plasticity model [16]. Acknowledging that the microstructure is not negligible when compared to the high stress gradients associated with fretting, the Dang Van high cycle fatigue parameter was investigated as an FIP. Although some comparisons with test data have been carried out in terms of crack location and orientation, previous work has not addressed microstructuresensitive life prediction for crack initiation, and hence fatigue life predictions, per se. In recent work, the authors [17,18] have presented a CP approach for prediction of fretting wear crack nucleation of Ti-6Al-4V and FF prediction of 316L stainless steel
(SS). The microstructure-sensitive model captured the location, orientation and numbers of cycles to crack initiation when compared against interrupted fretting wear test data of Ti-6Al$4 \mathrm{~V}$. Therefore, the methodology has subsequently been applied to a FF loading situation for 316L SS and has been extended to total life predictions. For engineering design against fretting, a key constraint is the computational overhead associated with modelling of realistic components. This is compounded by the apparent need for concomitant simulation of wear and fatigue damage evolution, particularly in design across a range of relative slip. The identification of slip regime (partial versus gross) is highly complex and dependent on coefficient of friction and contact geometry evolution, among other factors. Hence, whilst there is a requirement, on the one hand, for a scale-consistent accurate method for crack nucleation prediction, there is a pragmatic need, on the other hand, for robust, efficient methods and models for design [19]. This paper is concerned with a comparative assessment of microstructure-sensitive FF prediction and a $J_{2}$ plasticity methodology for FF crack nucleation and life prediction for 316L SS, in terms of (i) accuracy for crack nucleation and total life, vis-à-vis fidelity to test data, and (ii) numerical efficiency for engineering design. The paper presents specific new observations in relation to the predicted effects of grain orientation on FF crack initiation, particularly in the context of the interaction between surface grain size $(d)$, fretting stroke $(\delta)$ and contact (semi-) width $\left(a_{0}\right)$, as illustrated in Fig. 3, for example. In the present work, the ratios $a_{0} / d$ and $\delta / d$ are small in microstructural terms, leading to a significant predicted effect of grain orientation on FF life. 


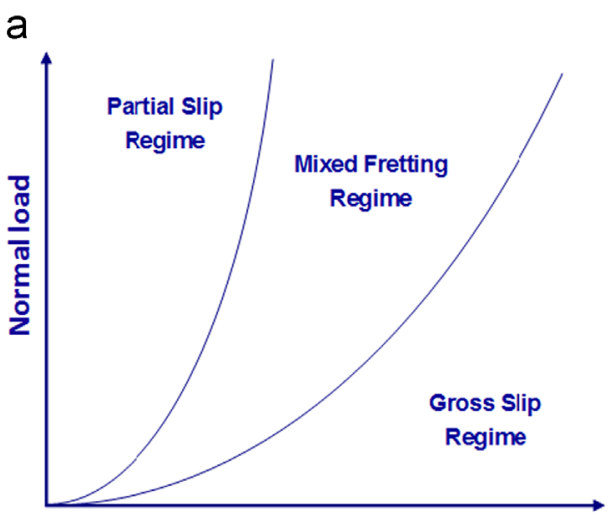

Displacement Amplitude

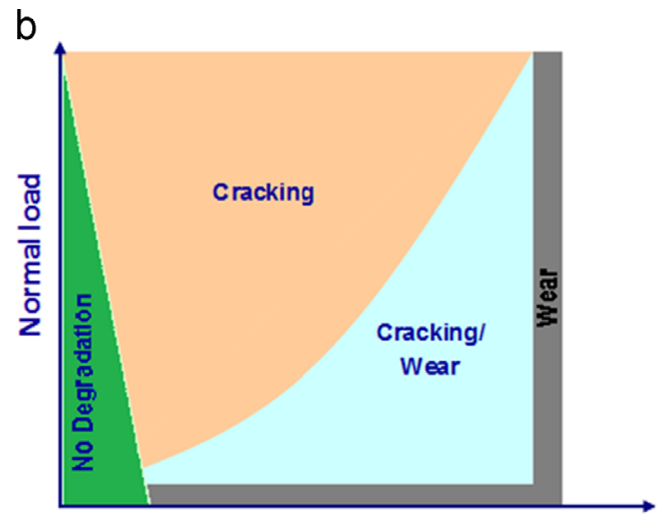

Displacement Amplitude

Fig. 1. (a) Running Condition Fretting Map (RCFM) and (b) Material Response Fretting Map (MRFM).

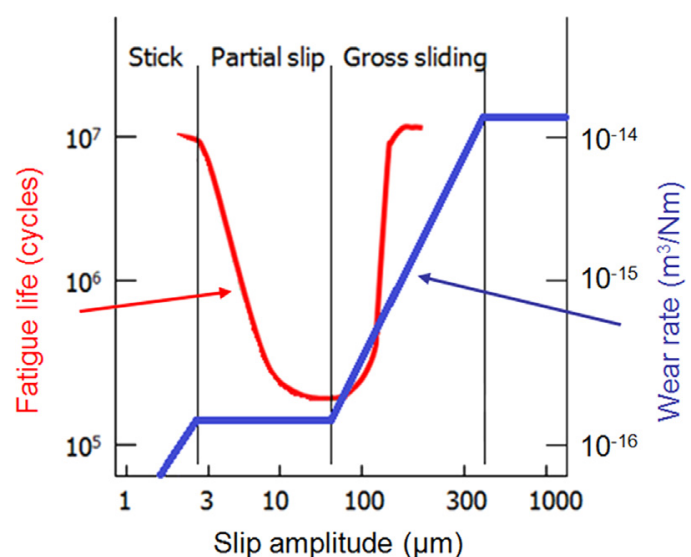

Fig. 2. Schematic illustration on the effect of slip amplitude on fatigue life and wear (after Vingsbo and Soderberg [8]).

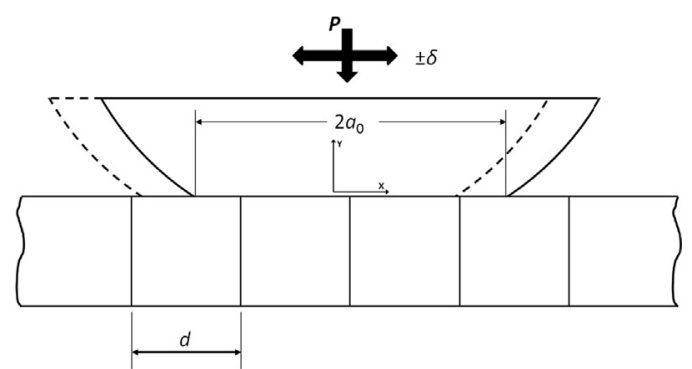

Fig. 3. Schematic highlighting the proportions of grain size $d$ to contact width $2 a_{0}$ and slip amplitude $\delta$.

\section{Methodology}

\section{1. $J_{2}$ NLKH model}

The $J_{2}$ plasticity formulation employed here incorporates nonlinear kinematic hardening (NLKH) to model the Bauschinger effect. The plastic flow-rule, defining the plastic strain increment is given by the following equations:

$d \boldsymbol{\varepsilon}^{p}=d \lambda \frac{\partial f}{\partial \boldsymbol{\sigma}}=\frac{3}{2} d p \frac{\boldsymbol{\sigma}^{\prime}-\boldsymbol{x}^{\prime}}{\sigma_{e}}$

$d p=\left(\frac{2}{3} d \varepsilon^{p}: d \varepsilon^{p}\right)^{1 / 2}$

where $f$ is the von Mises yield function, $d p$ is the increment in effective plastic strain, $\sigma_{e}$ is the von Mises equivalent stress, $\boldsymbol{\sigma}^{\prime}$ is the deviatoric stress tensor and $d \lambda$ is the plastic multiplier. $f$ is given as follows:

$f=\sigma_{e}-\sigma_{y}=\left(\frac{3}{2}\left(\boldsymbol{\sigma}^{\prime}-\boldsymbol{x}^{\prime}\right):\left(\boldsymbol{\sigma}^{\prime}-\boldsymbol{x}^{\prime}\right)\right)^{1 / 2}-\sigma_{y}$

where $\sigma_{y}$ is the yield stress and $\boldsymbol{x}^{\prime}$ is the deviatoric backstress tensor. The translation of the centre of the yield surface in kinematic hardening is governed by the backstress tensor $\mathbf{x}$, defined here via the Frederick-Armstrong non-linear hardening rule as follows:

$d \boldsymbol{x}=\frac{2}{3} C d \boldsymbol{\varepsilon}^{p}-\gamma \boldsymbol{x} d p$

where $C$ is the initial hardening modulus and $\gamma$ is the rate of decay of the modulus. The cyclic strain-hardening behaviour is given by the following equation [20]:

$\frac{\Delta \sigma}{2}-k=\frac{C}{\gamma} \tanh \left(\gamma \frac{\Delta \varepsilon_{p}}{2}\right)$

where $k$ is the cyclic (initial) yield stress. Identification of the NLKH parameters, $k, C, \gamma$ is achieved using Eq. (5), via a process described elsewhere, e.g. see [20]. This process uses the cyclic response of the material from strain-controlled tests at different strain-ranges, e.g. see [21].

\subsection{Crystal plasticity calibration}

The CP theory [22] used is a rate-dependant crystallographic theory that models the deformation of a metallic crystal lattice. It has been previously presented by the authors $[17,18]$, but is briefly outlined here for completeness, particularly for definition of the key constitutive parameters. Plastic slip is assumed to obey Schmidt's law [22], where the rate of plastic shear strain, $\dot{\gamma}^{\alpha}$, for a particular slip system, $\alpha$, is assumed to depend on the resolved shear stress, $\tau^{\alpha}$, through the following power law:

$\dot{\gamma}^{\alpha}=\dot{\operatorname{asgn}}\left(\tau^{\alpha}\right)\left\{\left|\frac{\tau^{\alpha}}{g^{\alpha}}\right|\right\}^{m}$

where $\dot{a}$ and $m$ are the reference strain rate and rate sensitivity exponent, respectively. In this work $m$ is chosen to be large enough to minimise the strain rate dependence of the equation. Material strain hardening on slip system $\alpha$, is specified by the slip system strain hardness, $g^{\alpha}$, which is defined by the integral of the following equation:

$\dot{g}^{\alpha}=\sum_{\beta} h_{\alpha \beta} \dot{\gamma}^{\beta}$

where $h_{\alpha \beta}$ is the strain hardness moduli and $\alpha$ and $\beta$ represent particular slip systems. The accumulated slip, $\gamma_{a}$ is defined as 
follows:

$\gamma_{a}=\sum_{\alpha} \int_{0}^{t}\left|\dot{\gamma}^{\alpha}\right| d t$

This theory is implemented here in the general-purpose, nonlinear FE code, Abaqus via a user-defined material UMAT user subroutine $[23,24]$.

Isotropic elasticity is assumed within the $\mathrm{CP}$ user subroutine for the 316L SS material, with a Young's modulus of $213 \mathrm{GPa}$ and Poisson's ratio, $\nu$, of 0.34 . The process of calibration of the $\mathrm{CP}$ constitutive constants, $h_{0}, g_{\infty}$ and $g_{0}$, for stress-controlled cyclic behaviour is similar to that described in [23] for strain-controlled cyclic behaviour. Essentially, a unit cell model of the material is developed, following the procedure described by Manonukul and Dunne [25] and the constants are identified, and presented in Table 1, via iterative comparison of the cyclic stress-strain hysteresis loops with the macroscopic cyclic, $J_{2}-\mathrm{NLKH}$, stress-strain curve for $316 \mathrm{~L}$ SS. The $J_{2}$-NLKH data is given in Table 2 [20]. This was corroborated for the material used here via comparison with the measured uniaxial tensile response of the material. As part of the calibration process, three different grain morphologies were considered, as shown in Fig. 4, namely a regular hexagon grain morphology (Fig. 4a), a Voronoi tessellation grain morphology (Fig. 4b) and a regular square grain morphology (Fig. 4c). This grain size is based on the experimentally obtained grain size of $19 \mu \mathrm{m}$ from optical microscopy of the etched 316L SS material microstructure (see McCarthy et al. [18]). In all cases, a random distribution of orientations was assigned to the grains, as described in [23]. The squares grains are attractive for the contact region of the fretting model, to allow better control of the contact region mesh, which is critical for the accurate computation of contact tractions and associated substrate stresses and strains, as well as crystallographic slip and slip system shear stresses. The square grain morphology was generated in a similar fashion to the hexagonal morphology, in terms of grain area, number of elements per grain, grain orientation and position. The square and hexagonal grain morphologies also included triple points, which are an important deformation and failure characteristic of real microstructures. Comparison of the $\mathrm{CP}$ stabilised cyclic stress-strain hysteresis loops, against the NLKH data of Table 2, for different strain-ranges has been presented in recent work [18]. The unit cell responses of the three morphologies showed negligible difference with respect to stressstrain response. Different (random) distributions of orientations were simulated for the square grain morphology in order to quantify the degree of scatter in the predicted unit cell response, both in terms of constitutive and failure behaviour

Table 1

Identified $\mathrm{CP}$ constitutive constants for cyclic behaviour of 316L SS.

\begin{tabular}{ll}
\hline$h_{0}$ & $10.5 \mathrm{GPa}$ \\
$g_{\infty}$ & $207.84 \mathrm{MPa}$ \\
$g_{0}$ & $82.68 \mathrm{MPa}$ \\
$\dot{a}$ & $0.0023 \mathrm{~s}^{-1}$ \\
$n$ & 30 \\
\hline
\end{tabular}

Table 2

Elastic and NLKH $J_{2}$ plasticity constants [20].

\begin{tabular}{ll}
\hline$E$ & $213 \mathrm{GPa}$ \\
$\nu$ & 0.32 \\
$k$ & $300 \mathrm{MPa}$ \\
$C$ & $30,000 \mathrm{MPa}$ \\
$\gamma$ & 60 \\
$K_{I c}$ & $40 \mathrm{MPa} \sqrt{m}$ \\
\hline
\end{tabular}

a

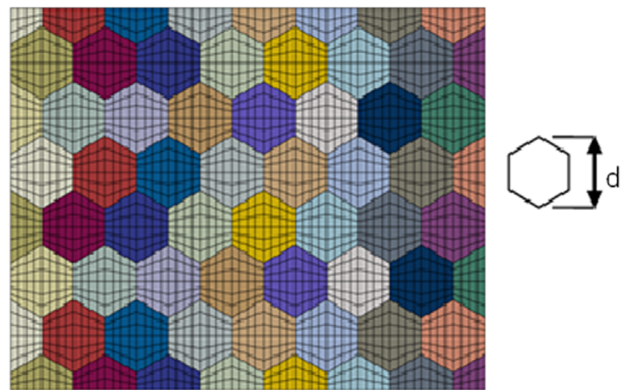

b

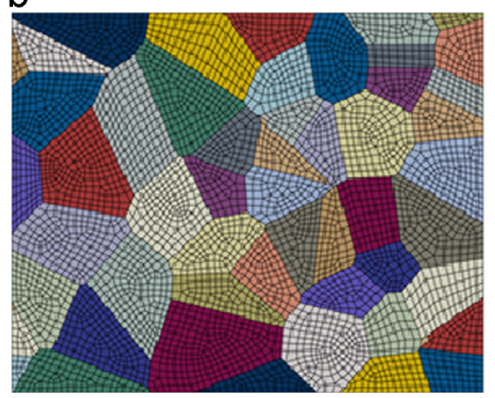

C

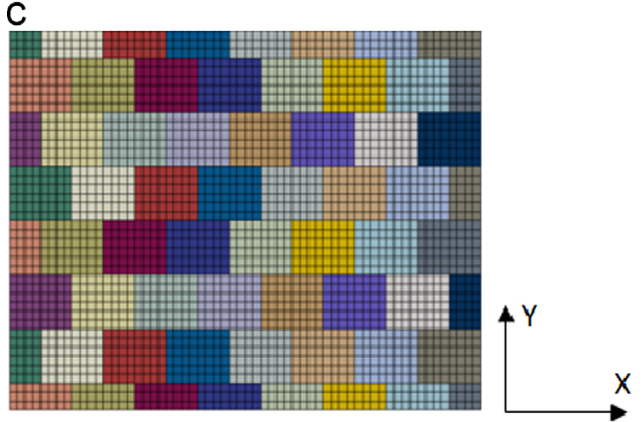

Fig. 4. Plane strain unit cell FE model for CP uniaxial simulations of 316L SS: (a) an assumed regular hexagonal grain shape and grain size dimension, $d$; see inset, (b) a Voronoi tessellation mesh of the microstructure, and (c) a square grain mesh.

\subsection{Crystal plasticity fatigue crack initiation (FCI) model}

For the $\mathrm{CP}$ model, it is necessary to employ a microstructuresensitive FIP for scale consistency. Manonukul and Dunne [25] were the first to present such a parameter, accumulated plastic slip, $p$, which was shown to have the ability, along with critical resolved shear stress, to predict both low and high cycle fatigue across a range of temperatures and mean stress levels for C263, a FCC nickel alloy. $p$ was argued to be the key microstructural parameter controlling crack initiation. Sweeney et al. [26] presented a study on the benefits of this approach for the micro-scale application of 316L stent fatigue, vis-à-vis a $J_{2}$ model with a Basquin-Goodman fatigue life prediction approach. A key benefit was the ability to predict scatter in fatigue life. $p$ is defined by the following equations:

$\dot{p}=\left(\frac{2}{3} \boldsymbol{L}^{p}: \boldsymbol{L}^{p}\right)^{1 / 2}$

$p=\int_{0}^{t} \dot{p} d t$

where the plastic velocity gradient $\boldsymbol{L}^{p}$ is defined by:

$\boldsymbol{L}^{p}=\sum_{\alpha=1}^{n} \dot{\gamma} \mathbf{s}^{\alpha} \mathbf{n}^{\alpha T}$ 
with $\mathbf{s}^{\alpha}$ and $\mathbf{n}^{\alpha}$ as the slip direction and normal vectors, respectively, for a given slip system, $\alpha$, with $n$ slip systems. The computation of $\dot{p}$ and $p$ is implemented within the CP user subroutine. Crack initiation is deemed to have occurred once $p$ reaches a critical value of accumulated plastic slip, $p_{\text {crit }}$. This critical value is calculated using an experimentally determined number of cycles to initiation, $N_{i}$, and the maximum (with respect to location) accumulated plastic slip in one cycle, $p_{c y c}$, for the same loading conditions. Due to the predicted rapid stabilisation of $p$ with respect to fatigue cycles, it is possible to determine $p_{\text {crit }}$ from the following:

$p_{\text {crit }}=N_{i} p_{\text {cyc }}$

This parameter has been further demonstrated (Sweeney et al. [27]) to successfully predict crack initiation location across a range of load cases, in comparisons with micro-structural experimental crack nucleation data from combined scanning electron microscopy (SEM) and electron backscattered diffraction (EBSD) for notched four-point bend fatigue tests and on FCC steel material.

\section{4. $J_{2}$ FCI damage model}

The Smith-Watson-Topper (SWT) parameter [28] is an example of a macro-scale FIP, which is thus normally considered to be a total life prediction parameter. Such 'total life' FIPs are based on calibration against experimental data corresponding to detection of surface fatigue cracks of about $1 \mathrm{~mm}$ in length [29]. In choosing an FIP for a given application it is important to consider suitability with respect to stress state and cracking behaviour, e.g. see $[5,30]$. Socie [29] makes recommendations regarding multiaxial FIP on the basis of mode of failure, controlled primarily by material behaviour and loading mode. For example, for Mode I (tensile) fatigue cracking, dominated by maximum principal stress (based on observed cracking directions relative to applied biaxial or multiaxial loading directions), SWT is recommended, while for Mode II failure (shear), dominated by shear fatigue failure, the Fatemi-Socie parameter (for example) is recommended. The plain fatigue (PF) testing of the present material exhibits Mode I (tensile) fatigue cracking. Hence, the SWT parameter is selected here for FCI prediction with the $J_{2}$ plasticity model. The SWT parameter is a combination of the Coffin-Manson and Basquin parameters for low and high cycle fatigue, respectively, multiplied by a $\sigma_{\max }$ term to account for mean stress effects as follows:

$S W T=\sigma_{\max } \frac{\Delta \varepsilon}{2}=\frac{\left(\sigma_{f}^{\prime}\right)^{2}}{E}\left(2 N_{f}\right)^{2 b}+\sigma_{f}^{\prime} \varepsilon_{f}^{\prime}\left(2 N_{f}\right)^{b+c}$

where $\sigma_{f}^{\prime}$ is the fatigue strength coefficient, $\varepsilon_{f}^{\prime}$ is the fatigue ductility coefficient, $b$ is the fatigue strength exponent, $c$ is the fatigue ductility exponent and $E$ is Young's modulus. This parameter has been adopted within a critical plane approach to allow multiaxial effects to be incorporated. This allows SWT to predict the "damage" per cycle on any given orientation. The critical plane implementation of Sum et al. [6], adopted here, calculates the SWT parameter by combining the peak normal stress $\sigma_{\max }$ and the strain range $\Delta \varepsilon$ within one cycle for a range of plane orientations at $5^{\circ}$ intervals through a $180^{\circ}$ range. Sum et al. [6] demonstrated successful application of the critical-plane SWT approach to elastic-plastic fatigue life prediction of a laboratory-scale spline coupling against test data. The critical-plane SWT has been used to produce realistic and accurate results compared to experimental data for FF of both Ti-6Al-4V [10,31] and CrMoV aero-engine steel [32], and in other work, such as Araujo and Nowell [5] and Szolwinski and Farris [33].

However, Madge et al. [34] highlighted an inconsistency in relation to the use of such an (FIP) approach for quantifying crack nucleation life and location. Since crack initiation, $N_{i}$, occurs at length scales of $10 \mu \mathrm{m}$ or below, and since FF stress gradient considerations (Sum et al. [6], Bernardo et al. [35]) lead to element integration points (or equivalent sample points, e.g. see [5]) typically at depths of $10 \mu \mathrm{m}$ or so from the surface, it is inconsistent to use SWT constants calibrated at about $1 \mathrm{~mm}$ crack length. Therefore, constants corresponding to $10 \mu \mathrm{m}$ cracking were back-calculated by Madge et al. [34] via a modified Paris equation, incorporating short crack growth (SCG) through an El-Haddad approach. A similar approach is used here to analytically back-calculate a crack initiation stress-life Basquin relationship from the experimentally determined PF stress-life data.

\subsection{Crack propagation model}

It is well known that short cracks propagate at a faster rate than long cracks [36]. To account for this phenomenon here, the El Haddad [37] methodology is applied. This approach incorporates a threshold crack length, $a_{t h}$, which demarcates the transition from SCG to Paris crack growth. The equation $a_{t h}=\frac{1}{\pi}\left(\frac{\Delta K_{t h}}{\sigma_{f l}}\right)^{2}$ is used to determine $a_{t h}$, where $\Delta K_{t h}$, is the threshold stress intensity factor (SIF) range for fatigue crack growth and $\sigma_{f l}$ is the stress fatigue limit. The El-Haddad correction is an empirical approach which allows for SCG prediction by substituting crack length, $a$, with $\left(a+a_{t h}\right)$ in the SIF range expression, when $a<a_{t h}$, as follows:

$\Delta K=\Delta \sigma Y \sqrt{\pi\left(a+a_{t h}\right)}$

In this work the modified Paris equation is used to calculate the number of cycles for a micro-crack to propagate, $N_{p}$, to failure, as follows:

$\frac{d a}{d N}=C(\Delta K)^{m}$

where $C$ and $m$ are short crack growth constants identified from experimental testing of 316L [38], as shown in Table 3. Once the fatigue stress-life data has been transformed to crack initiation stress-cycles data, it is possible to identify the Basquin constants corresponding to $1.2 \mu \mathrm{m}$ crack initiation, as follows:

$\frac{\Delta \sigma}{2}=\sigma_{f}^{\prime}\left(2 N_{i}\right)^{b}$

Crack propagation under FF conditions is a complex issue. Houghton et al. [32] implemented a weight function method, based on the work of Nicholas et al. [39]. This approach analysed the mixed-mode cracking of Ti-6Al-4V to successfully predict multiaxial FF life in a simplified representative FF test for spline coupling teeth. Once crack location was established local stress ranges were identified for Mode I and Mode II weight functions [40]. This approach allows incorporation of the effect of the contact-induced biaxial stress gradients on crack growth.

Table 3

Fatigue constants corresponding to and identified for $1.2 \mu \mathrm{m}$ nucleation crack length.

\begin{tabular}{ll}
\hline Constant & Value \\
\hline$\sigma_{f}^{\prime}$ & $869.4 \mathrm{MPa}$ \\
$\varepsilon_{f}^{\prime}$ & 0.54 \\
$b$ & -0.12 \\
$c$ & -0.495 \\
$a_{0}$ & $59.5 \mu \mathrm{m}$ \\
$\Delta K_{t h}[46]$ & $5.81 \mathrm{MPa} \mathrm{m}{ }^{0.5}$ \\
$\sigma_{f l}$ & $190 \mathrm{MPa}$ \\
$C$ & $2.0 \times 10^{-10}$ \\
$m$ & 1.9 \\
$p_{\text {crit }}$ & 37.73 \\
\hline
\end{tabular}


An effective stress intensity factor is defined as:

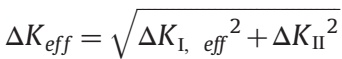

where

$\Delta K_{\mathrm{I}}$, eff $=\Delta K_{\mathrm{I}}(1-R)^{(1-n)}$

where $R>0$ and $n$ is the Walker constant $(0<n<1)$. Here, $n$ is chosen to equal 0.5 . If $R<0, \Delta K_{\mathrm{I}}=K_{\max }$, based on the Walker method [41]. $R$ is defined as $\sigma_{\min } / \sigma_{\max }$, where $\sigma_{\min }$ and $\sigma_{\max }$ are the minimum and maximum applied stress, respectively. The increment of crack growth $\Delta a$ is then calculated using a cycle-jumping approach as:

$\Delta a=C\left(\Delta K_{e f f}-\Delta K_{t h}\right)^{m} \Delta N$

where $\Delta N$ is the cycle jumping factor. The process was applied here for the $J_{2}$ plasticity and CP predicted nucleated micro-cracks to compute micro- (using the El-Haddad correction $a<a_{t h}$ with SCG constants) and macro-crack propagation across the width of the FF test specimen (substrate).

\subsection{Experimental testing}

A bridge-type FF test rig was designed and developed to characterise the effect of FF on 316L SS, as shown in Fig. 5. The design and development of this test rig has been described in detail in [18]. It is briefly described here. The test rig consists of a fretting bridge arrangement where each bridge, one on each side of the specimen, has two semi-cylindrical feet pressed onto the fatigue specimen via a proving ring, which maintains a constant normal load on the fretting pads. The fatigue specimen is cyclically loaded within an Instron servo-hydraulic testing machine. The cyclic strain in the fatigue specimen leads to relative displacement between the fatigue specimen and the fretting pads resulting in fretting, which combines with the substrate fatigue stress to generate $\mathrm{FF}$ in the substrate. A constant normal load of $0.5 P_{y}$ was applied in the tests, where $P_{y}$ is the normal load required to cause plasticity. PF tests, with $R=0.1$, were carried out to allow determination of the fretting fatigue reduction factor (FFRF), defined as the ratio of PF to FF life at a given cyclic stress amplitude. The maximum fatigue stress is higher than the yield stress of the material. Plasticity therefore occurs in the fatigue specimen during the first fatigue cycle, due to the $R=0.1$ stress ratio. This will shake down to elastic behaviour within the first few cycles. Table 4 shows the stress loading conditions used for the PF and FF tests, along with the measured numbers of cycles to failure for both the PF and FF tests. Repeat PF tests have been carried out for each stress level to give some measure of scatter. The measured ratios of standard deviation to mean

Table 4

Tabulated data of the experimental results for both plain and fretting fatigue over a range of $\sigma_{\text {amp }}$ values $(R=0.1)$. On average a fretting fatigue reduction factor of 3.5 is observed relative to the plain fatigue results.

\begin{tabular}{lllll}
\hline Test & Test type & $\sigma_{a m p}$ & $N_{f}$ & FFRF \\
\hline 1,2 & PF & 225 & 102284,112108 & \\
3,4 & PF & 213.75 & 113796,177862 & \\
5,6 & PF & 202.5 & 160816,279580 & \\
7 & PF & 191.25 & Run-off & \\
8 & FF & 225 & 56669 & $1.8-2.0$ \\
9 & FF & 213.75 & 59780 & $1.9-3.0$ \\
10 & FF & 202.5 & 35091 & $4.6-8.0$ \\
11 & FF & 191.25 & 245806 & $>12.2$ \\
\hline
\end{tabular}

Note: $\mathrm{PF}=$ plain fatigue; $\mathrm{FF}=$ fretting fatigue; FFRF range based on repeat $\mathrm{PF}$ tests.

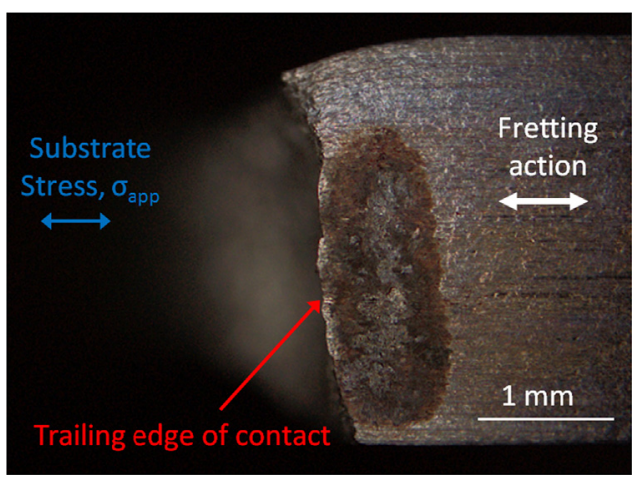

Fig. 6. Plan view of an optical image of a typical fretting wear scar highlighting the failure at the trailing edge of contact.

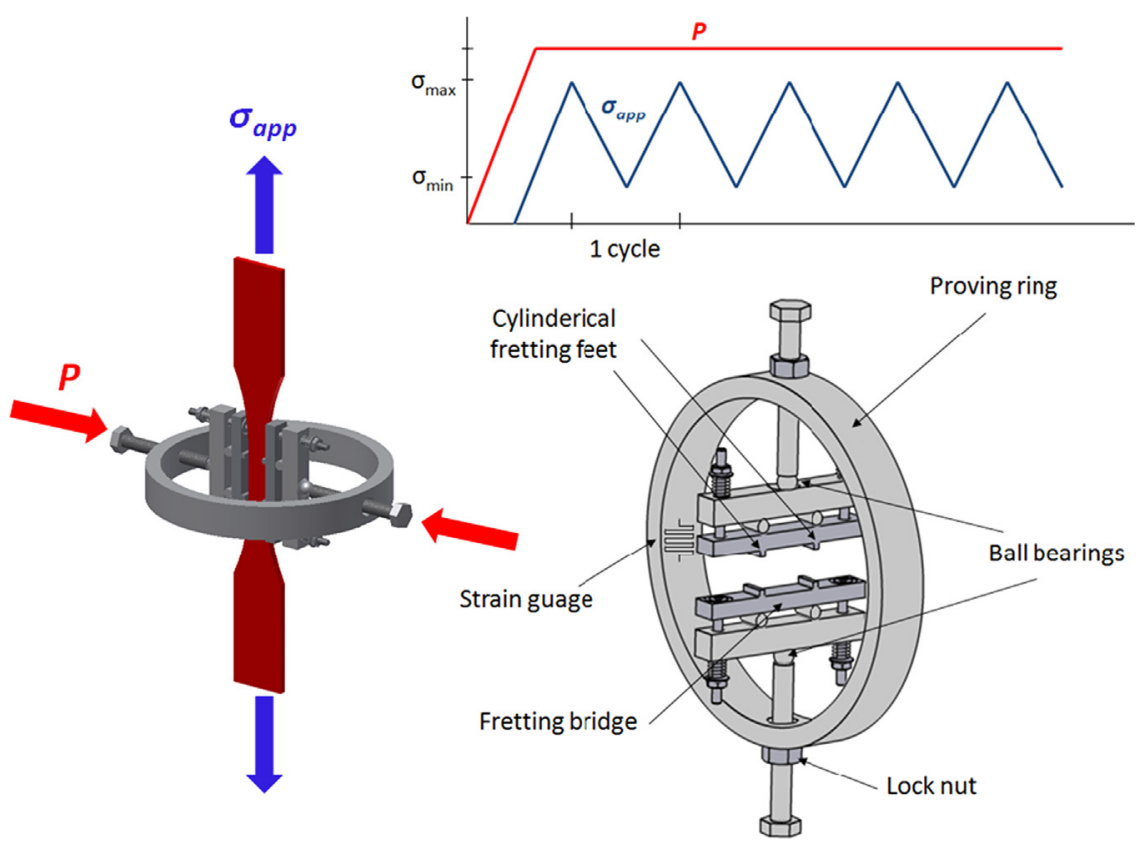

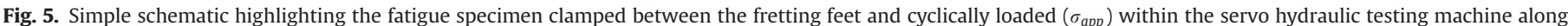
with the load history. 
$(\sigma / \bar{x})$ of PF life range from 0.06 to 0.38 , increasing with reducing stress level. The fretting cracks, in all cases, occurred on the outermost contact edges of the pair of fretting bridge feet (pads), which corresponds to the trailing edge of contact coinciding with the instant of maximum fatigue (cyclic) stress, i.e. the trailing edge with maximum tangential (surface) stress, due to the superposition of frictional stress and substrate fatigue stress. An example of such a crack is shown in Fig. 6. Although not shown here, SEM images of the plain fatigue cracks suggested crack initiation from the free edges of the fatigue specimen. In contrast, SEM images of the fretting fatigue cracks do not indicate initiation from the free edges, but rather from a point under the contact, in the wear scar, away from the edges [42].

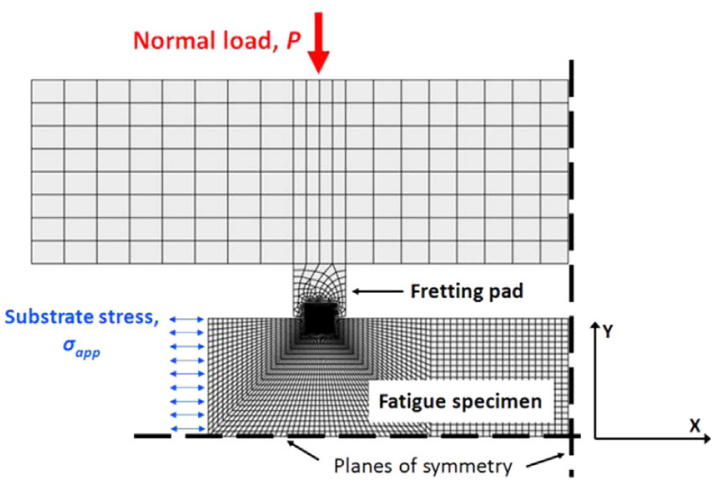

Fig. 7. FE model of one quarter of the fretting fatigue rig highlighting boundary constraints and applied loading.

\subsection{Fretting fatigue modelling}

The FE model of the fretting rig is a one quarter model of the complete rig, as shown in Fig. 7. The fretting contact is modelled as a 2D plane strain cylinder-on-flat fretting model. The radius of the cylindrical pad is $6 \mathrm{~mm}$, held in contact with the $4.5 \times 13.5 \mathrm{~mm}$ substrate under a fixed normal (clamping) load. The substrate represents one quarter of the gauge length with symmetry boundary conditions on the bottom and right edges. The substrate is subjected to the same cyclic loading conditions as in the experiments. Linear equation constraints are defined on the left edge of the substrate to ensure uniform nodal displacements in the horizontal $X$ direction between a master node and slave nodes when applying cyclic loading. The same method is used on the fretting pad to enforce uniform nodal displacements in the vertical $Y$ direction when applying the normal force $P . P$ is applied in the first step and held constant throughout the analysis. In the second and subsequent steps the cyclic loading is applied to the substrate to simulate the experimental conditions as shown in the loading history of Fig. 5. Generally, in fretting contact, as discussed in McColl et al. [43] for an unlubricated metallic steel contact, the COF increases from a low value of about 0.3 over the first thousand or so cycles to a significantly higher steady-state value, which depends on stroke, normal load and possibly other factors. In the present work a constant steady-state COF of 0.8 is adopted, based on the average value measured in [43]. A more detailed description of the FE frictional contact methodology is presented in McCarthy et al. [23]. The CP contact region is embedded within a $\mathrm{NLKH}_{2}$ plasticity bulk model. This CP region is 20 grains wide and

b
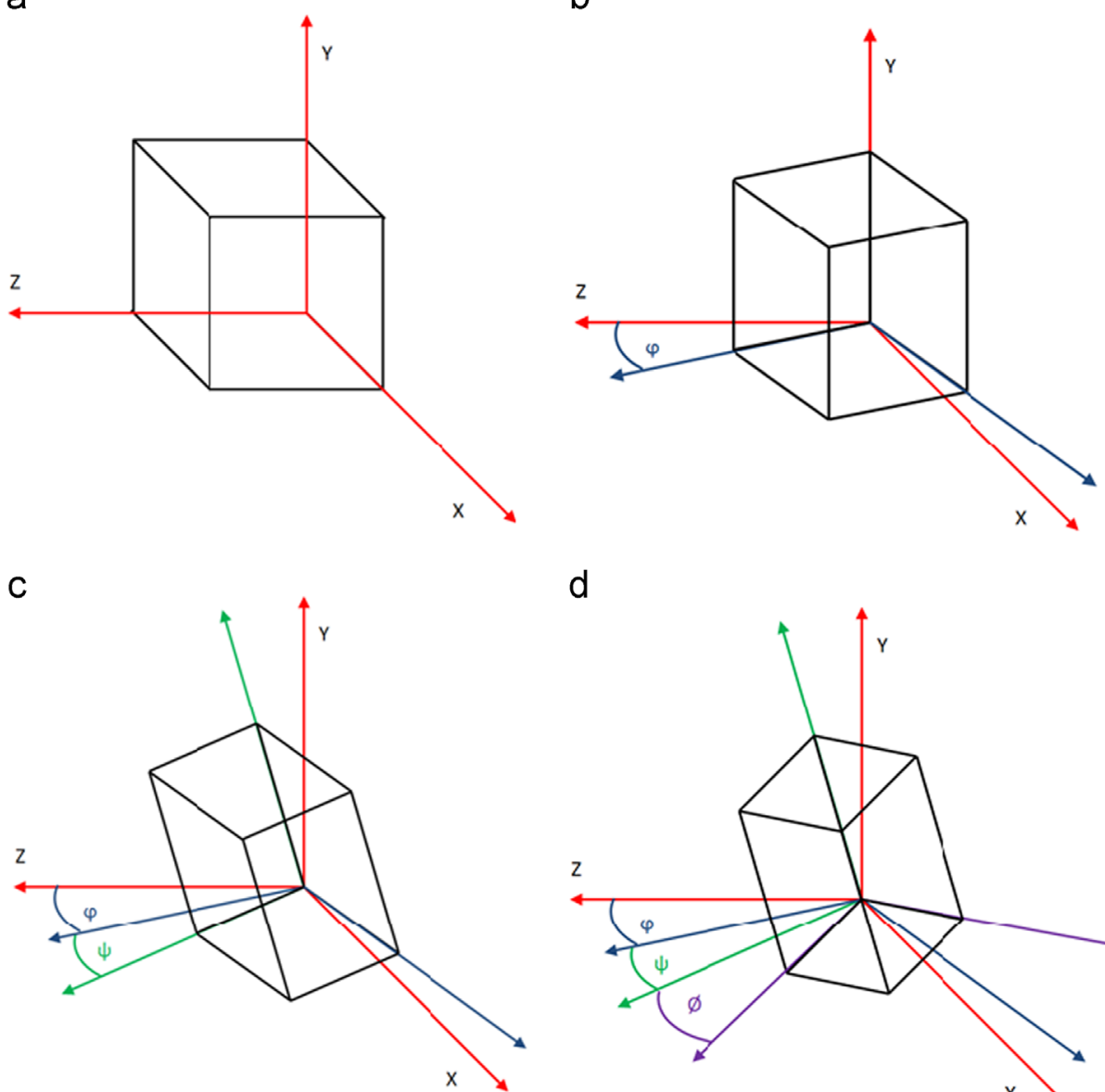

d

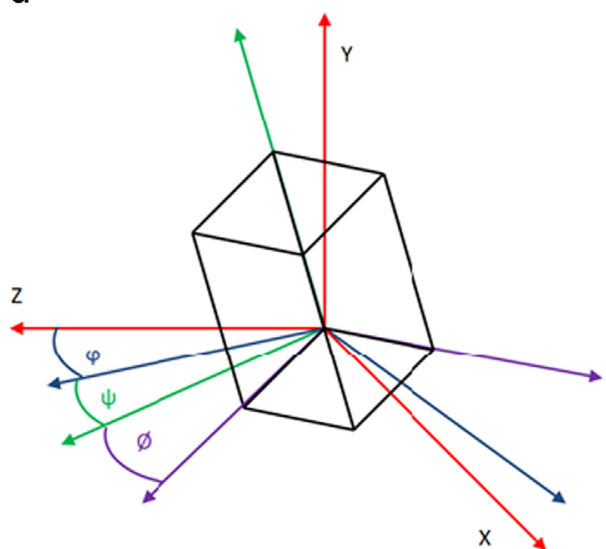

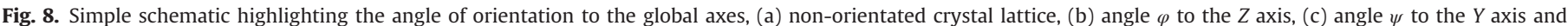
(d) angle $\varnothing$ to the $X$ axis. 
a

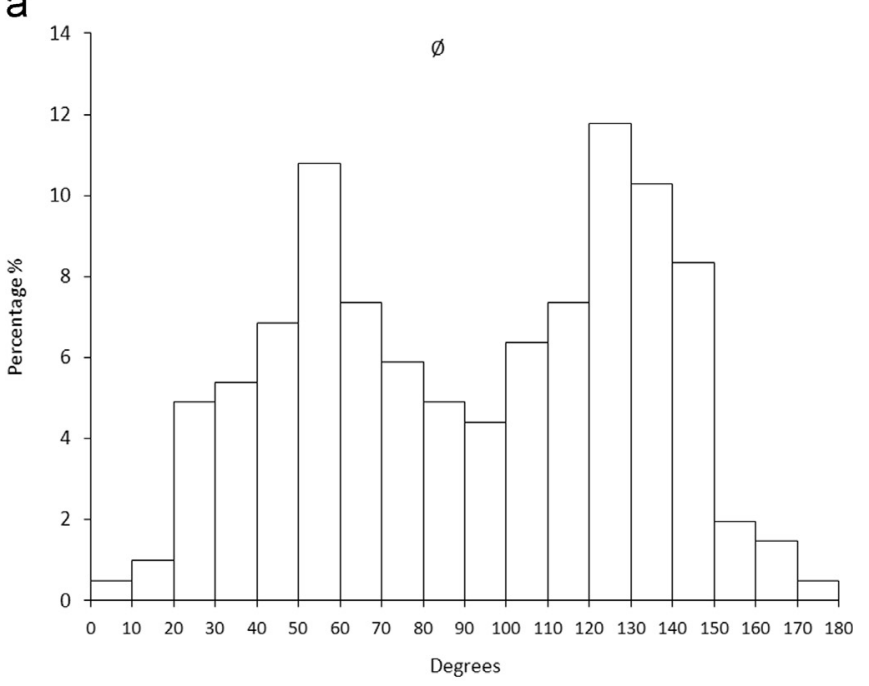

b

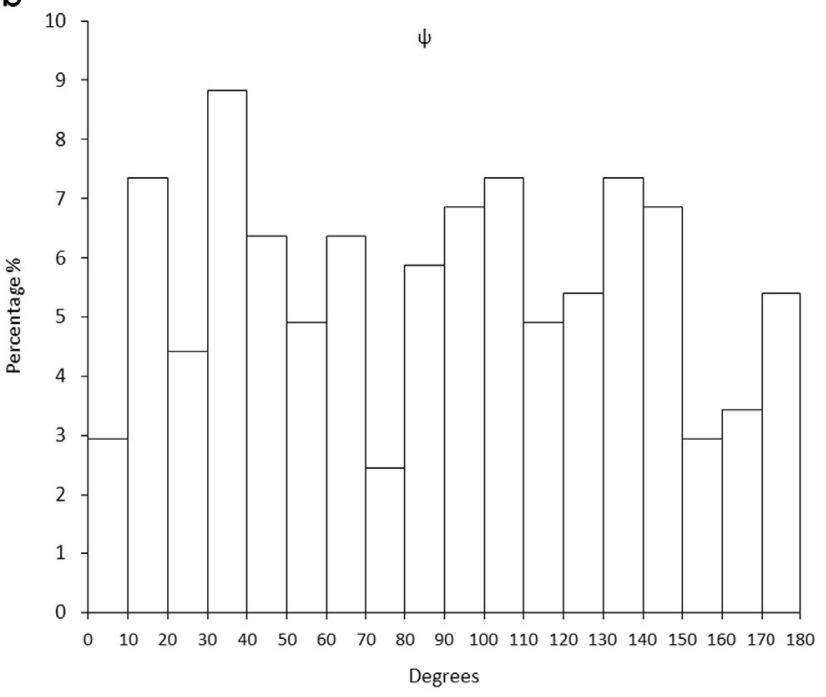

C

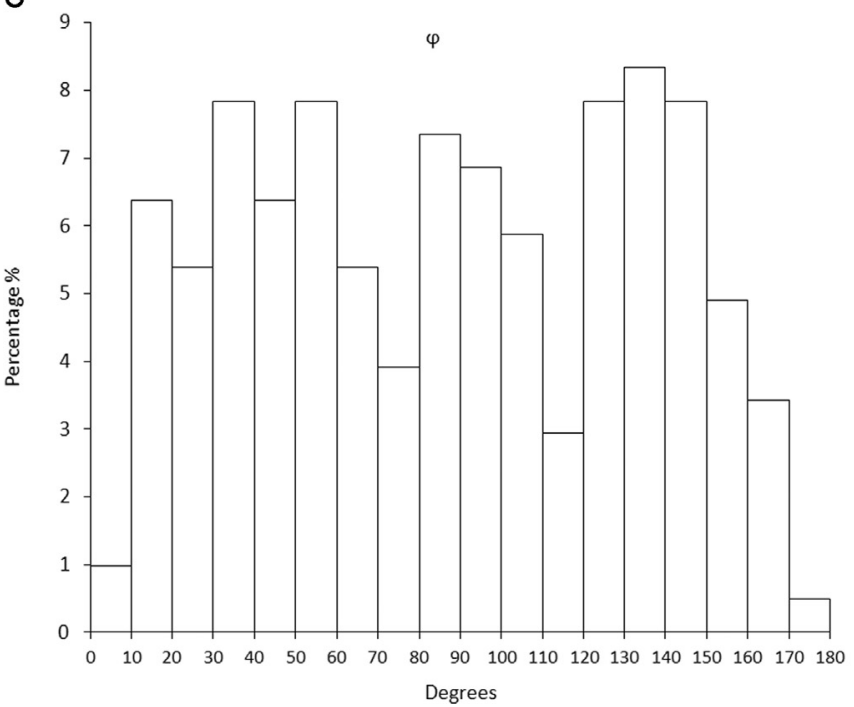

Fig. 9. Histograms representing the initial orientations of the crystal grains to the $X, Y$ and $Z$ global axis for the entire CP region of SET 2 .

10 grains deep. A square grain morphology is used to facilitate better mesh control, which is important for accurate resolution of surface and sub-surface fretting variables. An element size of
Table 5

Mean and standard deviation of angle $\varnothing$ (degrees) to the $X$ axes for five sets of random orientations.

\begin{tabular}{llllll}
\hline & SET-1 & SET-2 & SET-3 & SET-4 & SET-5 \\
\hline Mean & 81.6 & 91.9 & 89.9 & 88.8 & 88.1 \\
Stdev & 38.8 & 40.6 & 39.3 & 36.0 & 34.2 \\
\hline
\end{tabular}

$2.5 \mu \mathrm{m} \times 2.5 \mu \mathrm{m}$ is used in the contact region, with a decreasing mesh density further away from the $\mathrm{CP}$ region. 4 noded plane strain (CPE4) elements are used within this work. An important aspect of solution accuracy within CP modelling is element area to grain area ratio. Previous work by Harewood and McHugh [44] highlights the importance of this ratio for monotonic loading of stent struts. Converged results are presented for grains with a mesh to grain area ratio of $\sim 0.01$; in this work a ratio of $\sim 0.015$ is implemented. Previous convergence and mesh refinement studies were carried out in McCarthy et al. [23]; the current element type and mesh density are sufficient for converged surface and subsurface results. Furthermore, this approach is consistent with that of Sum et al. [6] to capture stress gradient effects, e.g. for contact size effects.

A random distribution of orientations is assigned to the grains in the $\mathrm{CP}$ region of the fretting model. The orientations can be specified in terms of angles of the rotated crystal to the original $X-Y-Z$ axis system, as illustrated in Fig. 8. In order to assess the effect of grain orientation on FF, five distributions of random orientations (SET 1 to SET 5) are considered, with simulations performed at all test stress amplitudes. As an example, Fig. 9 shows the histograms of distributions of grain orientations for SET 2 in the CP region of the hybrid model. It is clear that there is a random distribution of orientations. Table 5 presents data on the mean and standard deviation (Stdev) for all five random orientation sets for angle $\emptyset$, to the $X$ axis. The histogram distributions of grain orientations relative to the $Y$ and $Z$-axes follow a similar pattern. The five distributions of random orientations are thus seen to be nominally similar. Detailed CP results for sample sets, specifically, SET 1 and SET 2, are presented below and compared to the $J_{2}$ results. Life prediction results for all sets are presented to provide information on the scatter in FF life predicted by the microstructure sensitive model, for comparison with the measured data.

The NLKH $J_{2}$ plasticity FF model uses the same geometry, mesh and boundary conditions as the CPFE fretting model but the $\mathrm{CP}$ constitutive model is replaced by the NLKH $J_{2}$ plasticity formulation described in Section 2.2.

\subsection{Microstructure-sensitive fretting damage}

It is important, particularly in the context of micro-crack nucleation, due to contact-induced stresses, to develop a method for distinguishing between fretting wear and cracking. Combined wear-fatigue modelling, using a critical-plane SWT approach, for example, has been successfully applied to predict the effect of slip amplitude on FF life, specifically, the beneficial effect of gross slip wear, in terms of both contact stress re-distribution and reduction of crack growth rate [10]. Ding et al. [45] presented an alternative method, circumventing the need for explicit simulation of wearinduced material removal. This was achieved through the use of a FF damage parameter $D_{\text {fret } 2}$, for incorporating the effects of surface (fretting) damage within an FE-based multiaxial, critical-plane FIP. The FF damage parameter was defined as

$D_{\text {fret } 2}=(1+C \tau \delta)\left\langle 1-\frac{\tau \delta}{(\tau \delta)_{\text {th }}}\right\rangle^{n}$ 
a

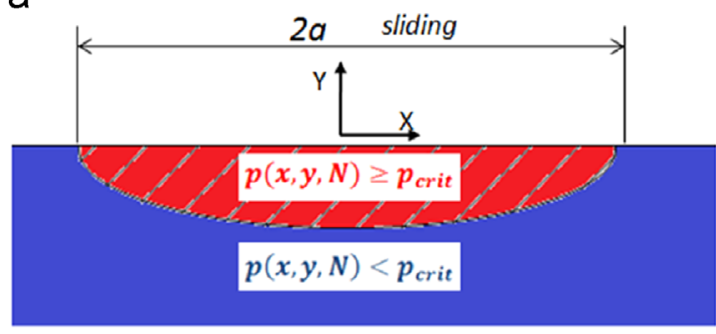

b

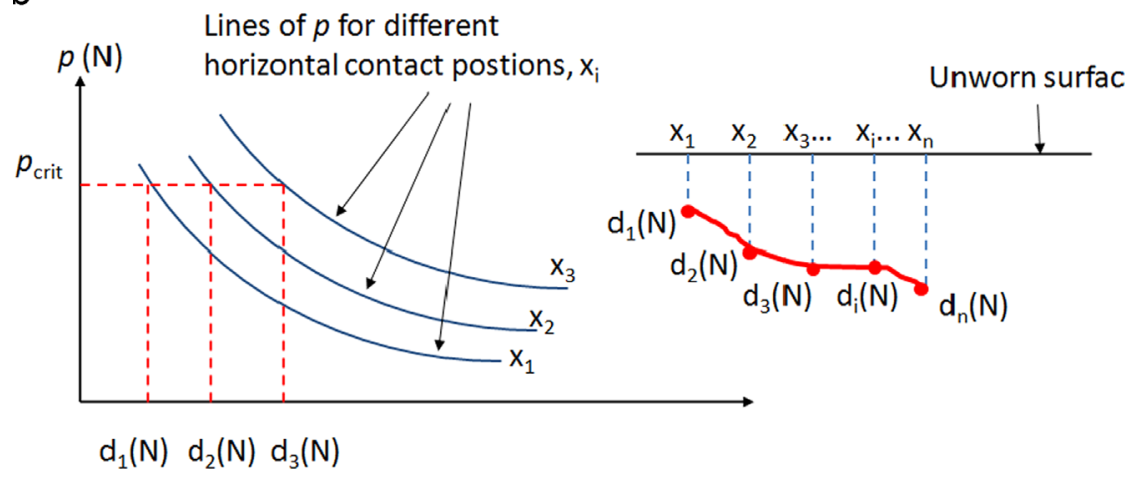

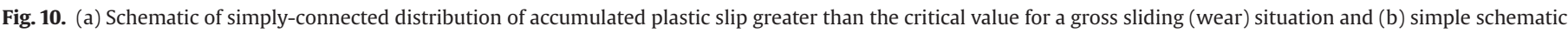
of the CPFE fretting wear predictive methodology for a given number of fretting cycles $N$.

where the symbol \langle\rangle is defined by $\langle u\rangle=u$ if $u>0$ and $\langle u\rangle=$ 0 if $u \leq 0$. The frictional work threshold value, $\tau \delta_{t h}$, separates cracking from a wear dominated fretting damage. $C$ and $n$ are constants determined from fretting tests. It was shown that the combined SWT- $D_{\text {fret } 2}$ method could simulate the characteristic beneficial effect of increasing (gross) slip amplitude on FF life, without computationally-expensive material removal simulations. It was argued in [45] that the surface damage parameter could be computationally determined using CP modelling of the rough surface damage.

Some success was achieved in [17], by the authors, in relation to prediction of FF crack nucleation and wear damage for both partial and gross slip loading conditions of Ti-6Al-4V [17] using the micro-cracking $p_{\text {crit }}$ approach, with application to fretting wear conditions, i.e. without the complication of a substrate fatigue load. Fig. 10 illustrates the fretting wear (micro-cracking) damage concept via a simple schematic. A gross slip wear scar is identified as a simply connected, evenly distributed region of accumulated crystallographic slip, $p$, greater than the critical value, $p_{\text {crit }}$. Sweeney et al. [26] have shown that cyclic micro-plasticity occurs in situations where loading is ostensibly elastic (at a macro scale). This distributed micro-cracking process, effectively wear, is characteristic of gross slip situations where micro-damage occurs throughout the entire contact region. The accumulated plastic slip per cycle (stabilised) parameter, $p_{c y c}$, is implemented here, within a numerical methodology to predict wear depth, based on the assumption that any material with $p(x, y, N) \geq p_{\text {crit }}$ has been worn away after that number of fretting cycles, $N$, as illustrated in Fig. 10. Further details are given in [17].

\section{Results}

\subsection{Contact and FCI variable distributions}

As discussed above in relation to the $D_{\text {fret2 }}$ parameters for surface fretting damage, the local distributions and evolutions of contact tractions and relative slip are important for prediction

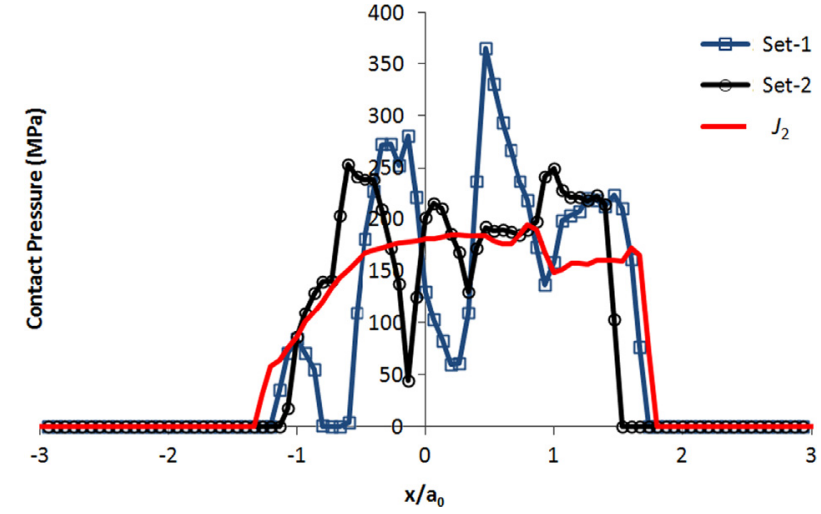

Fig. 11. Predicted CP (SET 1 and SET 2) and $J_{2}$ fretting model (stabilised) distributions of contact pressure for $\sigma_{a m p}=213.75 \mathrm{MPa}$ at instant of maximum applied substrate load.

Table 6

Initial $(N=0)$ contact semi widths for analytical and FE models.

Contact semi width, $a_{0}(\mu \mathrm{m})$ Maximum contact pressure, $p_{\mathrm{o}}(\mathrm{MPa})$

$\begin{array}{lll}\text { Hertzian } & 33 & 322 \\ \text { CPFE } & 36 & 320 \\ J_{2} & 36 & 324\end{array}$

of fretting wear and damage. These variables are also critical to the interpretation and hence application of simplified laboratory test configurations to more complex, realistic (application) geometries, e.g. see Ratsimba et al. [19]. Furthermore, it is of interest to assess how the CP tractions and slip evolve with localised microplasticity, with a view to wear simulation via micro-plasticity. The CP- and $J_{2}$-predicted evolutions (with fretting cycles) of contact variable distributions are presented in Fig. 11 (contact pressure) and Fig. 12 (shear traction); the CP distributions shown correspond to grain orientation distributions of SET- 1 and SET 2 (see Table 5). Table 6 shows a comparison of the CP and $J_{2}$ contact 
semi-widths and pressures against the Hertzian (theoretical) values, clearly showing correlation. The most obvious observation in relation to these surface traction distributions is the contrast between the CP- and $J_{2}$-predictions. The initial $(N=0)$ contact width is only about four grains wide (i.e. $a_{0} / d \sim 2$, see Fig. 3 ), so that the $\mathrm{CP}$-predicted distributions of contact tractions are therefore highly inhomogeneous, particularly when compared to the $J_{2}$-predictions. Significant widening of the contact region is predicted by both models within the first tangential cycle, to a width of about six grains. This is attributed to surface plasticity effects, due to the high $\operatorname{COF}(=0.8)$ and the large relative slip to contact semi-width ratio $\left(\delta / a_{0} \sim 0.5\right.$, see Figs. 3 and 13). The applied fatigue loads are sufficient to cause plasticity in the fatigue specimen, but this shakes down elastically after the first cycle due to the $R=0.1$ substrate fatigue stress ratio. The mechanical behaviour of the surface of the CP model is controlled by the (random) crystallographic shear strength and slip behaviour of the four to six grains in the contact region, which are of course also anisotropic. It should be noted that the distributions for $N>1$, for both models, are sampled at the instant of maximum fatigue stress in the fatigue (fretting) cycle. Thus the $N>1$ distributions of both the CPand $J_{2}$ models are non-symmetric, with a clear re-distribution bias to the $+x$ direction (instantaneous leading edge). The shear traction distributions, due to the predicted gross slip regime, are proportional to the contact pressure, the constant of proportionality being the COF. For the $\mathrm{CP}$ predicted distributions, there are significant deviations from the $J_{2}$ (homogeneous, isotropic) distributions. For example, the steadystate shear traction for SET 2 at $x \approx-0.2 a_{0}$ is less than $30 \%$ of the $J_{2}$ prediction, while the $x \approx-0.7 a_{0}$ value (close to the instantaneous trailing edge of contact) is significantly larger, approximately $167 \%$ of the $J_{2}$ prediction. The CP (SET 1 ) and $J_{2}$-predicted contact slip distributions of Fig. 13 illustrate the predicted gross slip nature of the slip regimes. In both cases, the widening of the contact region on the first application of tangential shear (1st FF cycle) leads to contact edge regions of highly non-uniform slip. These regions experience intermittent contact. The CPFE plasticity model predicts inhomogeneity of contact slip also, with dramatic local reductions of as much as $45 \%$, at $x=-0.8 a_{0}$, the location of maximum $p_{c y c}$ (see below). This is important in the context of the key (and complex) role of slip (see Figs. 1 and 2 and the discussion about $D_{\text {fret } 2}$ above) in fretting surface damage and, hence, crack nucleation. The predicted evolutions and distributions of the $p_{c y c}$ (SET 1 and SET 2) and critical-plane SWT parameters along the contact surface (depth of $1.2 \mu \mathrm{m}$ ) are presented in Figs. 14 and 15. Fig. 14 shows that, as for the surface tractions, the $\mathrm{CP}$-predicted distributions of FCI parameter exhibit significant inhomogeneity, due to the small number of grains in the contact region. For both plasticity models, the peak FCI locations are close to the $x<0$ edge of contact, which corresponds to the instantaneous trailing edge of contact at the instant of maximum applied fatigue (substrate) load.

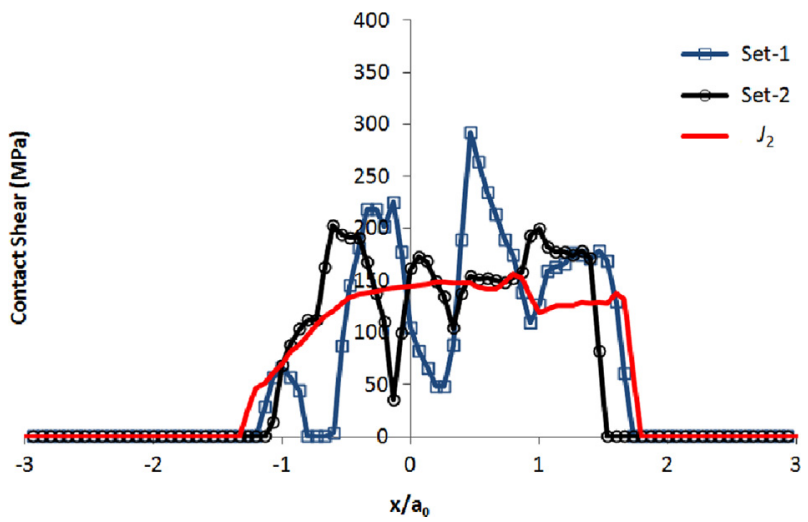

Fig. 12. Predicted CP (SET 1 and SET 2) and $J_{2}$ fretting (stabilised) distributions of contact shear for $\sigma_{a m p}=213.75 \mathrm{MPa}$.
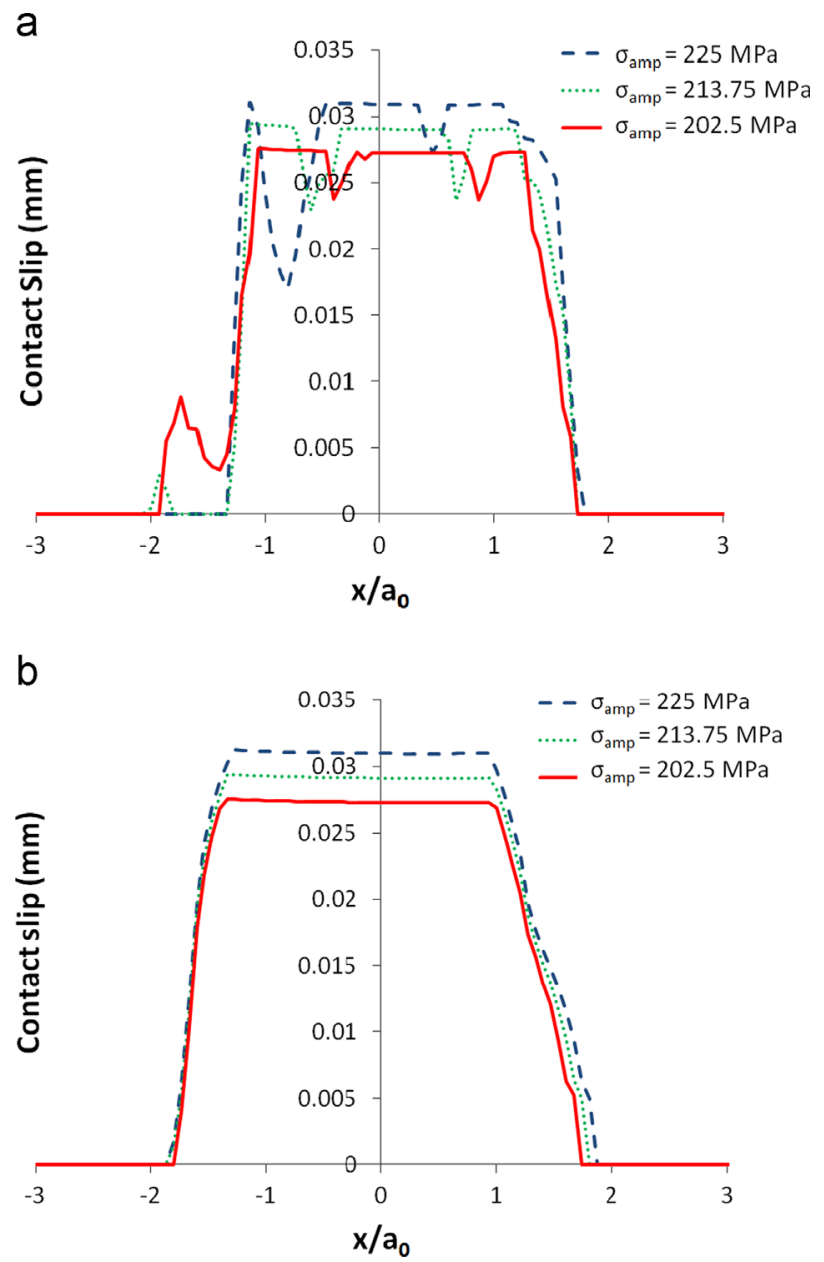

Fig. 13. Predicted effect of fatigue stress amplitude on distributions of relative (contact) slip for CP (SET 1) and $J_{2}$ fretting models. (a) CP model and (b) $J_{2}$ model.

As shown in (Fig. 6). The $\mathrm{CP}$ model predicts initiation (peak $p_{c y c}$ ) location at $x \approx-0.5 a_{0}$, i.e., inside the initial contact width at a grain boundary with non-uniform local slip and significant peak in shear and normal traction, whereas the $J_{2}$ model predicts FCI at $x \approx-1.6 a_{0}$, near the slip-induced edge of contact. The $J_{2}-S W T$ prediction can be thought of as equivalent to a $\mathrm{CP}$ case where there is a large number of grains (hence, homogeneous and isotropic) in the contact region. In all cases, the distributions of $p_{c y c}$ and SWT reduce with increasing $N$, but have approximately stabilised after 6 cycles, as shown in Fig. 14 for the 213.75 MPa fatigue stress. Fig. 15 shows the predicted effect of stress amplitude on the distributions of $p_{c y c}$ and SWT. Stress amplitude is predicted to have negligible effect on SWT but a significant effect on both magnitude and location of peak $p_{c y c}$. Specifically, with reducing fatigue stress, the peak $p_{c y c}$ value decreases and moves inward under the contact.

\subsection{Calibration of $p_{\text {crit }}$ and SWT}

In order to calibrate the microstructure sensitive $\mathrm{FCI}$ parameter, it is necessary to identify a critical value of $p_{c y c}$, as per Eq. (11). Using the values of $\Delta K_{t h}$ [36], threshold crack length, $a_{t h}$, and fatigue limit, $\sigma_{f l}$, listed in Table 3 , as well as the SCG constants $C$ and $m$ from [38], based on short crack growth testing of 316L SS, it is possible to back-calculate the numbers of cycles for propagation $\left(N_{p}{ }^{\exp }\right)$ of a $1.2 \mu \mathrm{m}$ crack to failure, defined here as a length of $1.5 \mathrm{~mm}$. Hence it is possible to infer $N_{i}{ }^{e x p}$ from the experimental PF 
a

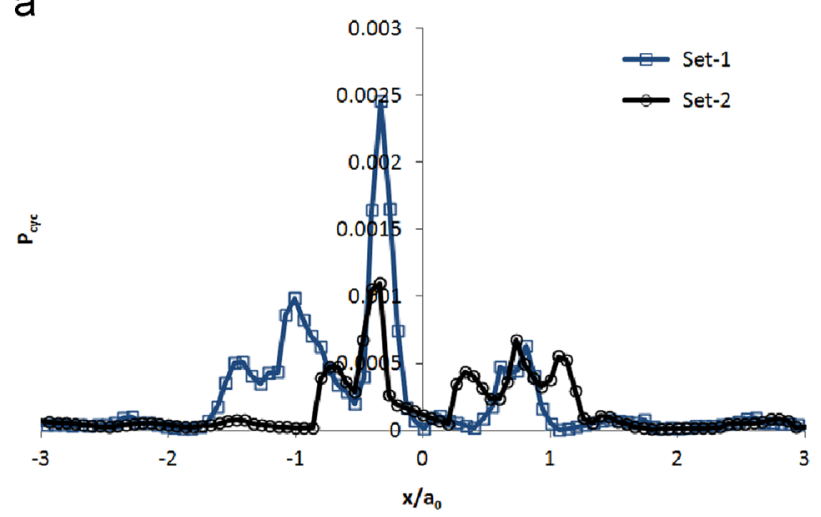

b

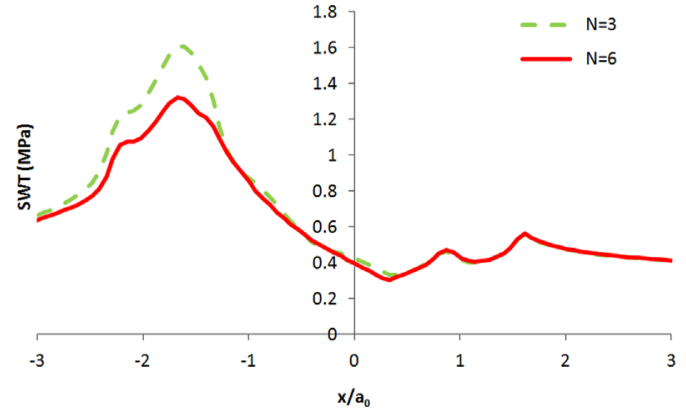

Fig. 14. Predicted CP (SET 1 and SET 2) $p_{c y c}$ and $J_{2}$ FIP distributions across the contact surface for $\sigma_{a m p}=213.75 \mathrm{MPa}$. (a) CP (SET 1 and SET-2) model, $\sigma_{a m p}=213.75 \mathrm{MPa}, N=6$ and (b) $J_{2}$ model, $\sigma_{a m p}=213.75 \mathrm{MPa}$.

stress-life data, as follows:

$N_{i}^{e x p}=N_{f}-N_{p}{ }^{e x p}$

$N_{p}{ }^{e x p}$ is found to be relatively small, only about $15-30 \%$ of $N_{f}$.

It is then possible, to identify the value of $p_{\text {crit }}$ as follows:

(1) Choose one (sample) stress amplitude and identify the associated $N_{i}{ }^{e x p}$ value.

(2) Identify the mean value of the CP-predicted $p_{c y c}$ values for this stress amplitude, by statistical analysis of the unit cell CPFE stress-controlled cyclic responses for a range of different (random) grain orientation distributions.

(3) Eq. (11) then provides the $p_{\text {crit }}$ value using the results from Steps 1 and 2 .

(4) Then validate the identified $p_{\text {crit }}$ value against the CPFE predicted unit cell response at other stress amplitudes, again using the mean response from a range of different (random) grain orientation distributions.

(5) In the present work, the identified value of $p_{\text {crit }}$ is 37.73 , as shown in Table 3. Fig. 16 shows a comparison of the resulting CP-predicted stress-cycles to crack initiation response and the $N_{i}{ }^{e x p}$ data. The CP-predicted ratio of standard deviation to mean $(\sigma / \bar{x})$ of cycles to PF nucleation (initiation) is 0.25 to 0.34 , depending on stress level; this is similar to that of the measured PF (total) lives.

A similar approach is used to identify the nucleation-scale $(1.2 \mu \mathrm{m})$ Basquin constants from the mean $N_{i}{ }^{\text {exp }}$ stress-cycles data and Eq. (15), as also shown in Fig. 16. The $\varepsilon_{f}^{\prime}$ and $c$ values were then iteratively chosen to achieve a good fit of SWT to the PF response, $N_{i}^{\text {exp }}$. The identified SWT constants are listed in Table 3. a
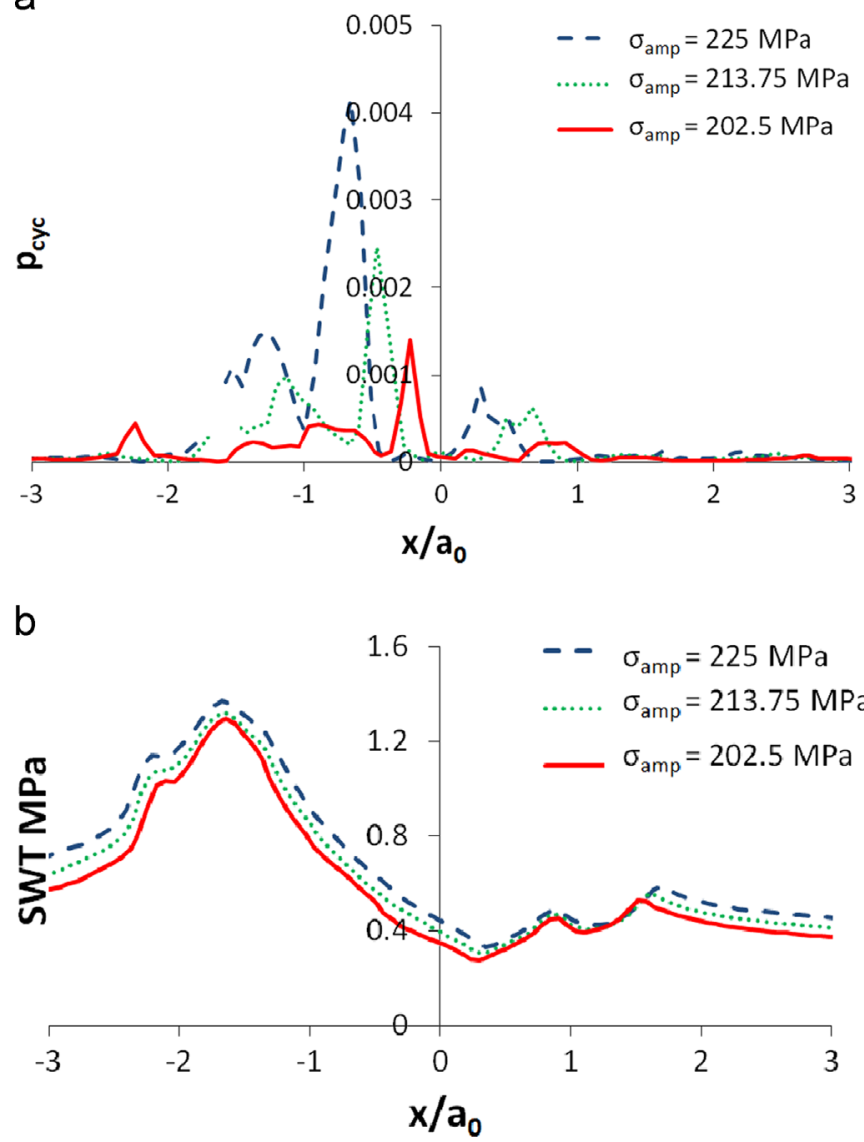

Fig. 15. Predicted effect of fatigue stress amplitude on stabilised (after 6 fretting cycles) contact distributions of (a) $p_{c y c}$ (SET 1) and (b) SWT. (a) CP model and (b) $\mathrm{J}_{2}$ model.

\subsection{Fretting fatigue test results}

The reduction in fatigue life due to fretting is significant, relative to the scatter in PF life. Fig. 17 shows that the FFRF is seen to increase significantly with decreasing stress amplitude, from about 2 at the highest stress level to more than 12 for the lowest stress level. Hence the effect of fretting increases significantly with decreasing stress level. The effect of stress amplitude on FF life is seen to be negligible for the two highest stress levels, but there is a significant decrease in FF life with further decreasing stress amplitude leading to a significant increase in FFRF to between 4.6 and 8 (depending on PF test datum) at 202.5 MPa. At the lowest stress level, the PF results gives runout $\left(>3 \times 10^{6}\right)$, and although the FF test gives an increased FF life relative to the higher stress amplitude (which appears to give a reduced effect of fretting, due to the long PF life), this, in fact, corresponds to a significantly increased FFRF value of 12.2. Hence, it is clear that there is a significant effect of fretting on the fatigue life of 316L SS and this effect is seen to be most pronounced for lower stress levels (higher life). This is, of course, consistent with the fact that FF is generally considered to be a high cycle fatigue phenomenon. A key challenge in designing against $\mathrm{FF}$ is assessment of the relative effects of (i) slip amplitude, viz. surface damage (fretting) effects, on the one hand, and (ii) multiaxial fatigue, due to the superposition of substrate (uniaxial) fatigue and (frictional) contact-induced (multiaxial) stress concentrations, on the other hand. The present work is specifically concerned with gross slip conditions. Fig. 18 shows a schematic illustrating these two effects separately and the combined response. Previous experimental, e.g. Jin and Mall [9], and matching computational results, i.e. Madge 


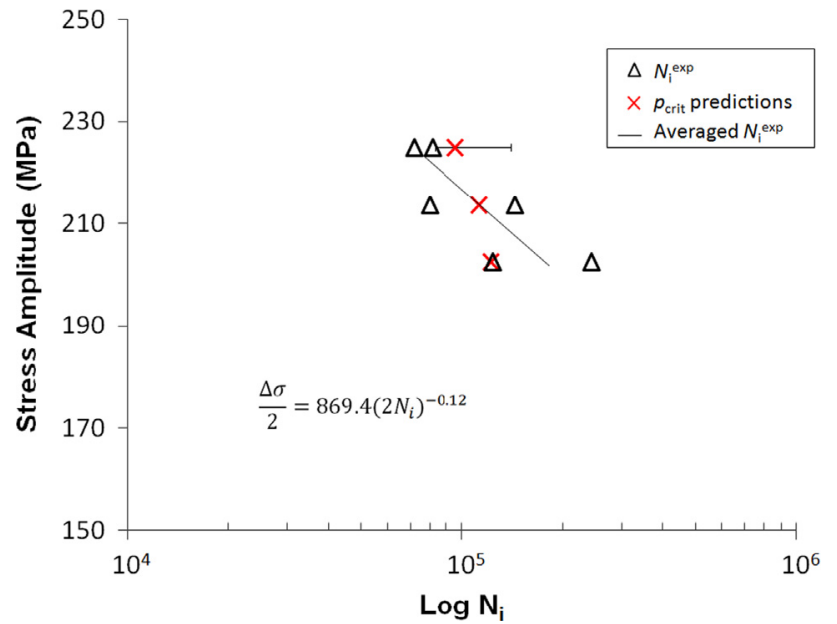

Fig. 16. Comparison between experimental and $\mathrm{CP}$ based predictions for number of cycles to crack initiation, $N_{i}$ for plain fatigue.

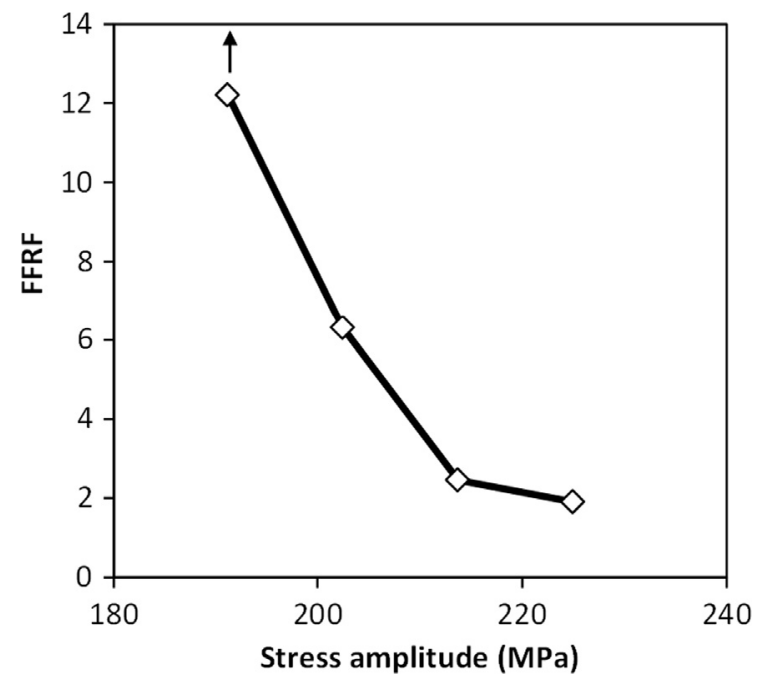

Fig. 17. Measured effect of stress amplitude on fretting fatigue reduction factor:

et al. [10], on the effect of slip (i), have shown that gross slip life can be expected to increase or level off with increasing stress amplitude (i.e. increasing slip), beyond a certain threshold, e.g. $\tau \delta_{t h}$, which is higher than the gross slip threshold. In contrast, the effect of (ii) is clearly to give decreasing life with increasing (substrate) stress amplitude in the gross slip regime, where trailing edge stresses have saturated beyond the gross slip threshold. These two cases represent boundaries of an envelope of possible interactive $\mathrm{FF}$ responses, with the more complex curve of Fig. 18 showing another possible characteristic response, whereby life initially decreases with reducing stress amplitude, due to pre-dominance of the fretting (surface) effects due to high relative slip, and then increases with further lowering of stress amplitude, due to the dominance of effect (ii), for example. This more complex effect corresponds to that measured experimentally in this paper for FF, although clearly the FFRF continues to increase with decreasing stress amplitude.

\section{4. $C P$ and $\mathrm{J}_{2}$ predicted fretting fatigue}

For the CP model and $J_{2}$ models, nucleated micro-cracks were assumed to propagate normal to the substrate surface, using the crack propagation methodology described above. This is consistent with the experimental observations whereby the FF cracks were

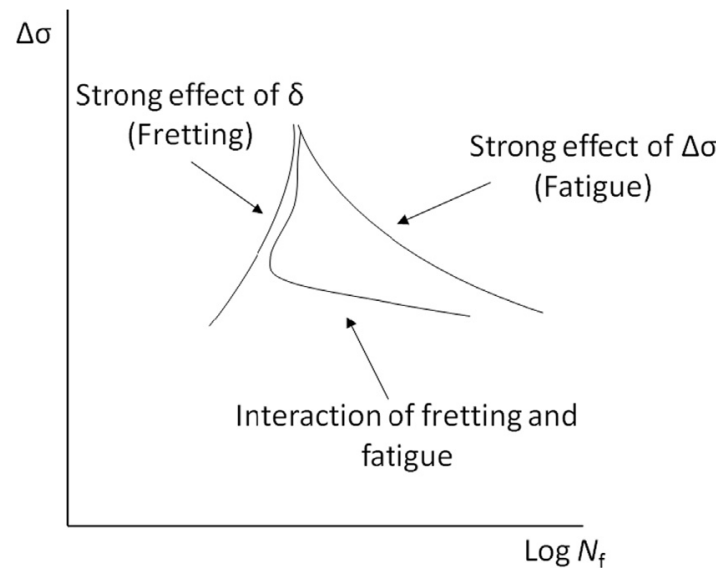

Fig. 18. Schematic illustration of the effects of fretting, fatigue and fretting-fatigue interaction in terms of the characteristic stress-life material response.

found to propagate at between $70^{\circ}$ and $110^{\circ}$ to the substrate surface. Sample predicted near-surface distributions of the relevant stresses driving micro-crack propagation are shown in Fig. 19 for both the $J_{2}$ and CP fretting models. Although not shown here, Negative $R$ ratio values are predicted near the contact surface. Therefore, the micro-cracks are subject to compressive loading at some point during the cycle. Fig. 20 illustrates the predicted micro- to macro-crack propagation across a range of $\sigma_{a m p}$ values for the $\mathrm{CP}$ fretting model (SET 1 orientation distribution). The critical failure length, $a_{c}$, based on the maximum stress, $\sigma_{\max }$, is calculated through the following equation:

$a_{c}=\frac{1}{\pi}\left(\frac{K_{I c}}{\sigma_{\max }}\right)^{2}$

where $K_{I c}$ is the fracture toughness, estimated here to be $40 \mathrm{MPa}$ $\sqrt{m}$ based on observed combinations of applied fatigue stress range and critical crack size. Different critical crack lengths are thus calculated for the different stress ranges.

In contrast to the PF situation described above, a significant proportion of fretting specimen life involves crack propagation. The predicted initiation $\left(N_{i}\right)$ and total $\left(N_{i}+N_{S C G}+N_{p}\right)$ lives for the $J_{2}-$ SWT and CP microstructure-sensitive approaches (SET-1) are compared in Fig. 21. Comparison with the CP and $J_{2}-\mathrm{SWT}$ predictions for PF gives in a CP-predicted (mean) FFRF of about 2.15 and a value of 1.5 for the $J_{2}-\mathrm{SWT}$ approach. The CP prediction is more consistent with the measured values of Fig. 17 particularly at the higher stress levels. The CP-predicted total life for SET 1 in Fig. 21 is accurate (relative to the test data) for the two highest stress levels; for the third highest stress level, the CP over-predicts life (non-conservative), while for the lowest stress level, the $\mathrm{CP}$ under-predicts life relative to the test result. In contrast, the $J_{2}$-SWT approach under-predicts for the two highest stress levels, over predicts for the third highest level (but is closer than the $\mathrm{CP}$ model) and under-predicts for the lowest stress level, by significantly more than the $\mathrm{CP}$ model. Hence, in terms of accuracy, the $\mathrm{CP}$ model is superior to the $J_{2}-\mathrm{SWT}$ approach at three of the four stress levels and, as shown below, the inferior performance of the $\mathrm{CP}$ model for the other stress level is directly attributable to the particular random grain orientation of SET 1; a different grain orientation for the $\mathrm{CP}$-predicted crack nucleation location can give lower life, thus reconciling the discrepancy with the test result. In terms of engineering design requirements for conservatism, it can be argued that the $J_{2}$-SWT approach is superior, due to being conservative in three cases and only slightly non-conservative in the fourth case. In terms of computational overhead, one $\mathrm{CP}$ fretting analysis required $\sim 972$ CPU hours (on a 36-core highperformance processor) whereas the $J_{2}$ fretting analysis only 
a

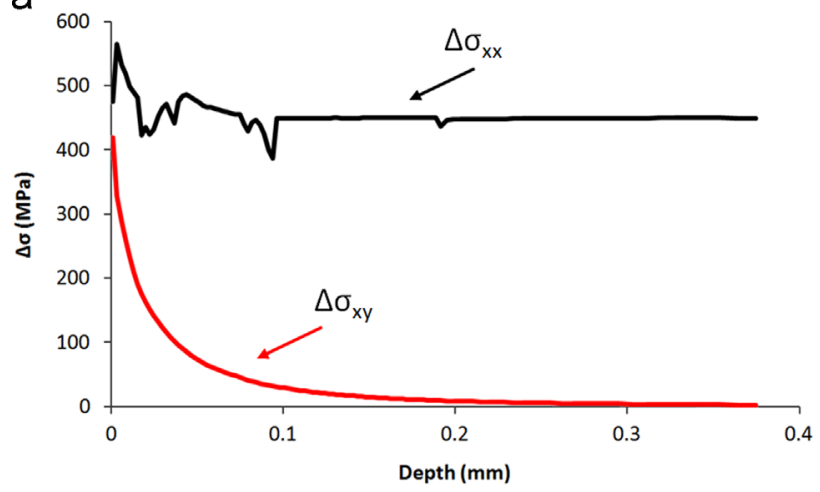

b

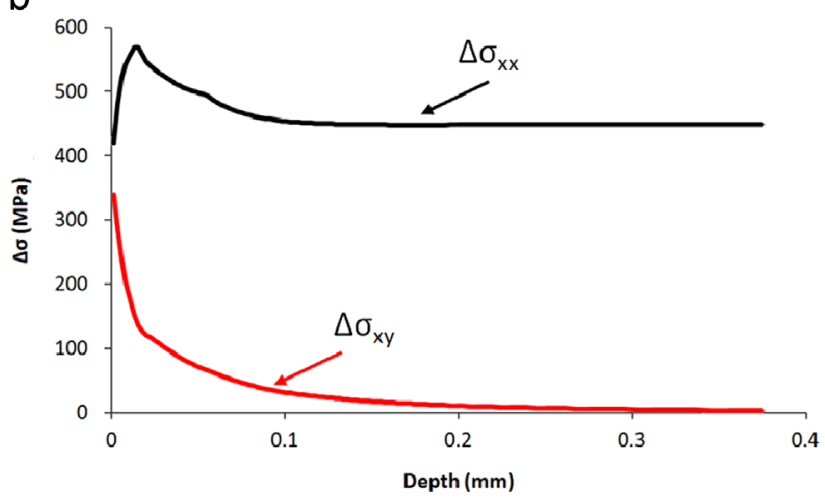

Fig. 19. Predicted distributions of $\Delta \sigma_{x x}$ and $\Delta \sigma_{x y}$ (stress amplitude parallel and perpendicular to the loading direction) stress gradients with depth into substrate (fatigue specimen) for (a) the CP model (SET 1) and (b) the $J_{2}$ model at $\sigma_{\text {amp }}=225 \mathrm{MPa}$.

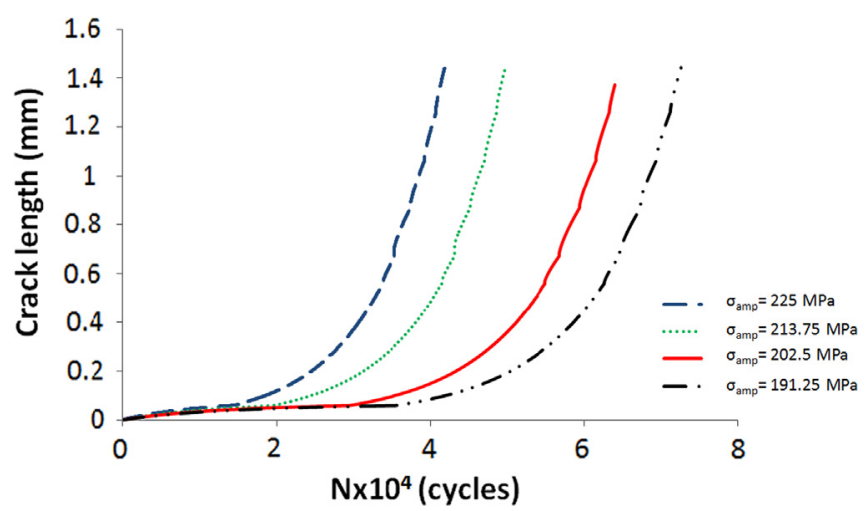

Fig. 20. Predicted effect of fatigue stress amplitude $\left(\sigma_{a m p}\right)$ on crack propagation for the CP model (SET 1).

required $\sim 11 \mathrm{CPU}$ hours on a 4-core computer. Each $\mathrm{CP}$ unit cell analysis required $\sim 18 \mathrm{CPU}$ hours on a 4-core computer.

\subsection{Effect of random orientation distribution on crack nucleation}

Fig. 22 shows a comparison of (i) the $\mathrm{CP}-p_{c y c}$ predictions (for all five distributions of random orientations) of FF crack nucleation life, (ii) the $J_{2}$-SWT FF crack nucleation life and (iii) the measured FF test total (nucleation and propagation) life, versus stress amplitude. The result of the crystallographic orientation study carried out using the five sets of randomly generated orientations assigned to each grain in the FE model are used to quantify scatter. Although the $J_{2}$ model does predict a significant effect of fretting (FFRF $>0$ ) relative to PF life, it predicts a negligible effect of fatigue stress amplitude, whereas the experiments and the $\mathrm{CP}$ model predict discernible effects of stress

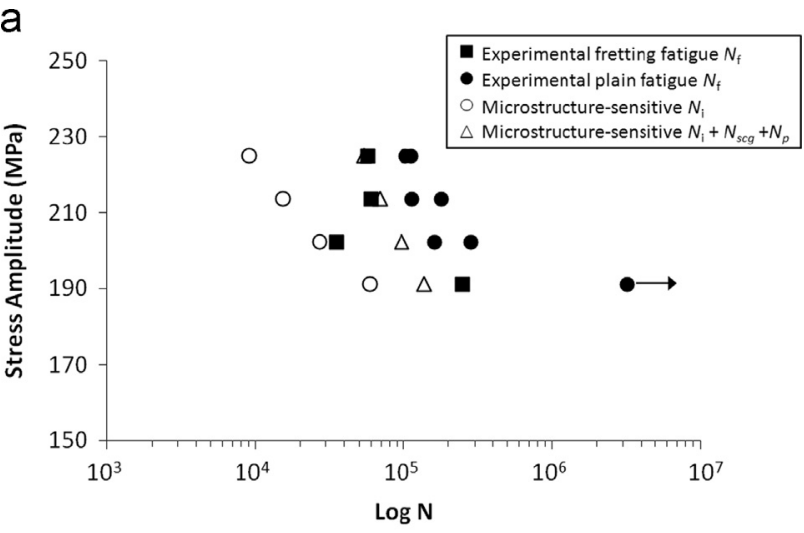

b

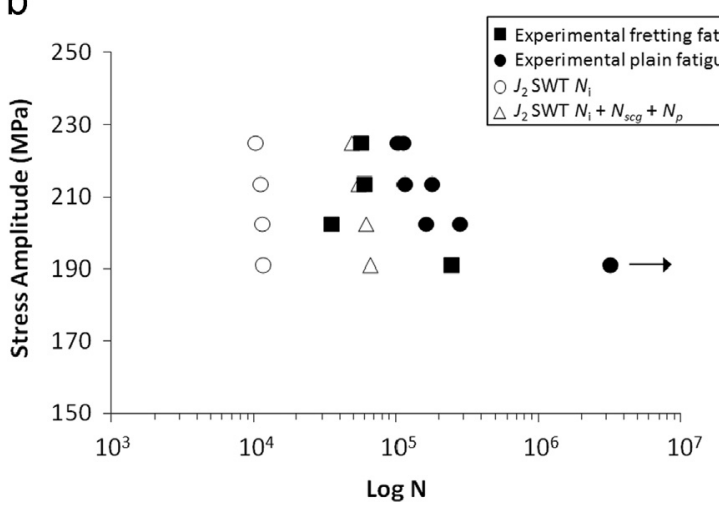

Fig. 21. Comparison of predicted initiation and total lives with experimental total lives for (a) CPFE model (SET 1) and (b) $J_{2}-$ SWT model.

amplitude. The lack of sensitivity of the $J_{2}$ model to stress amplitude, and hence slip amplitude, is entirely consistent with the anticipated result, due to the fact (e.g. see Madge et al. [10]) of saturation of shear traction (the key driving factor for trailing-edge tensile stresses) above the gross slip threshold, regardless of slip amplitude. The trailing-edge tensile stresses are, in turn, the key driving factor for FF crack nucleation. In contrast, it is clear that the typical CP response (e.g. SET 1) does predict both a significant effect of fretting and an effect of stress amplitude which captures the general measured trend of increasing life with decreasing stress (the exception here is at $202.5 \mathrm{MPa}$, where the fretting test gives a reduction in life with decreasing stress; this is discussed further below). An important observation from the CPFE results of Fig. 22 is the large range of $\mathrm{CP}$ predicted results. The $\mathrm{CP}$-predicted $\sigma / \bar{x}$ ratio for FF nucleation is between 0.8 to 1.2 , depending on stress level; this is significantly larger than the corresponding PF ratio, which indicates a prediction of significantly greater microstructure (grain orientation) sensitivity for FF than for PF. This is attributed here to the small $a_{0} / d$ ratio (small number of grains in the contact region) and small $\delta / d$ ratio (small contact slip to grain size ratio) and the assumed random orientation of these grains. This will, of course, make the crack nucleation response of trailing-edge material highly dependent on (random) grain orientation, hence leading to a significantly larger micro-structure sensitivity of FF crack nucleation. Indeed, the highly localised concentrations of $p_{c y c}$ in the small number of contact region grains suggests that computational efficiency could be significantly enhanced by embedding a reduced $\mathrm{CP}$ region (consisting only of grains in the contact region) in a larger homogeneous $J_{2}$ region. This approach would also potentially allow for the modelling of realistic grain geometries and 3D grains (McGarry et al. [47]) and for the simulation of crack propagation using the extended finite element method (Feerick et al. [48]). Additionally, as shown in the current study, grain orientation significantly affects the 
prediction of fretting fatigue. Given the critical contribution of plastic slip in a small number of grains at the specimen surface, experimental characterisation of the actual orientation of these grains could be performed in future studies e.g. You et al. [49], significantly improving on the accuracy of the $\mathrm{CP}$ predictions.

The following observations are made in relation to the individual CP random orientations:

(1) The predicted effect of random orientation distribution on nucleation life is significant, with SET 3 and SET 4 giving the lowest predicted lives and SET 1 and SET 2 giving the highest predicted lives (depending on stress amplitude).

(2) The differences in stress-life responses for each random distribution of grain orientations are also quite significant; SET 3 and SET 5 show weak sensitivity to stress level, akin to the $J_{2}$-SWT response. The SET 5 response is also quantitatively close to the $J_{2}-$ SWT model. In contrast, SET 1 gives a strong sensitivity to stress level while SET 4 shows a medium sensitivity to stress level.

(3) The (nucleation) stress-life response of SET 2 shows a qualitatively similar trend (although less pronounced) with respect to stress level (and hence relative slip level) to the measured (total) life response. Specifically, there is an overall increase in life with decreasing stress level, but there is a decrease in life between $213.75 \mathrm{MPa}$ and $202.75 \mathrm{MPa}$.

The test results for each stress level shown in Fig. 22 have been obtained from different test specimens. The grain distributions in each test specimen are different. For the purposes of comparison with the test results, it is reasonable to consider different random distributions of grains for different stress levels (specimens). Hence, by observation of the effect of grain orientation distribution on CP-predicted nucleation stress-life response, Fig. 23 shows a curve entitled 'selected random' which assumes SET 2, SET 1, SET 5 and SET 2, respectively, for the four test stress levels in descending order. This curve has an identical trend of stress-life response to the measured test data, thus demonstrating the ability of the $\mathrm{CP}-\mathrm{p}_{c y c}$ approach to represent complex (measured) FF slip-stress-life interaction relationships. Furthermore, both the $J_{2}-\mathrm{SWT}$ and mean $\mathrm{CP}-p_{c y c}$ responses, shown in Fig. 23, appear to be good approximations to a mean response for this curve.

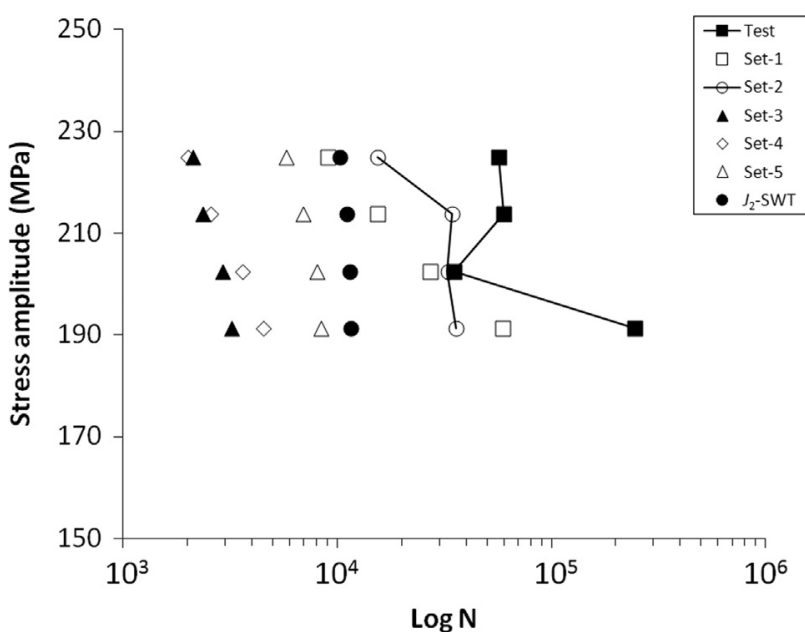

Fig. 22. Comparison of the fretting fatigue test data against the $\mathrm{CP}$ crack initiation predictions for 5 different random orientation sets and the $J_{2}-\mathrm{SWT}$ initiation predictions.

\subsection{CP-predicted wear}

Of course, an additional key challenge in relation to FF life prediction, is the development of a fundamental, micro-scale plasticity methodology for wear prediction, without recourse to measured wear profiles to identify wear coefficients, (e.g. see Fouvry et al. [50] and Ding et al. [51]), as input to wear simulation code for material removal. As discussed in Section 2.8, the CP- $p_{c y c}$ methodology for micro-crack prediction provides a basis for this, the wear mechanism being identified as a distributed microcracking process. This methodology has been applied here to the $225 \mathrm{MPa}$ stress amplitude FF test to predict a two-dimensional wear scar. Fig. 24 shows a comparison of this wear scar with a sample measured profilometry trace from the same test, after 56,669 cycles. The Archard wear coefficient is given as:

$k=\frac{V}{P S}$

where $k$ is the wear coefficient, $V$ is the measured wear volume, $P$ the normal load and $S$ is the total sliding distance. It is instructive to identify this wear coefficient from the CPFE wear prediction. In order to identify a value based on the measured wear scar, it is necessary to estimate relative slip, which has not been measured here. The CPFE predicted slip is used here for this purpose. The resulting 'experimental' value is $5 \times 10^{-8} \mathrm{MPa}^{-1}$ while the CPFE-predicted wear coefficient $0.5 \times 10^{-8} \mathrm{MPa}^{-1}$. A value of $1.5 \times 10^{-8} \mathrm{MPa}^{-1}$ was reported by Hutchings for stainless steel [52]. Clearly, the CPFE wear coefficient is in reasonable agreement, given the level of uncertainty around wear coefficient estimation (e.g. Ratsimba et al. [19]). Some additional observations can be made in relation to this CPFE-predicted wear result (Fig. 24):

(1) It is interesting to observe the W-shaped geometry of the predicted wear scar. Measured gross slip fretting wear scars are commonly U-shaped but some authors have observed W-shaped scars for gross slip conditions, e.g. [53-55]. However,

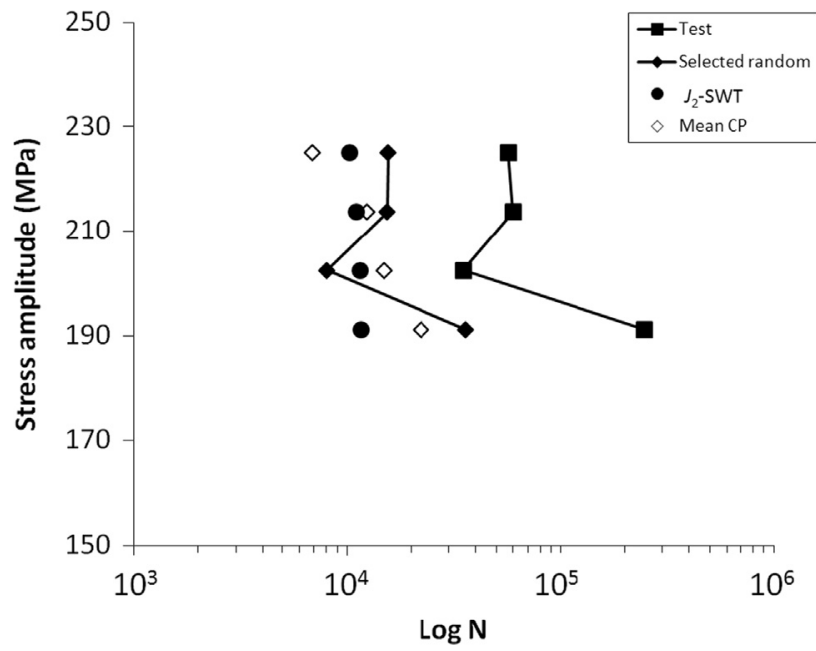

Fig. 23. Comparison of the selected random orientations, mean CP and the $J_{2}-\mathrm{SWT}$ initiation predictions against the fretting fatigue test data.

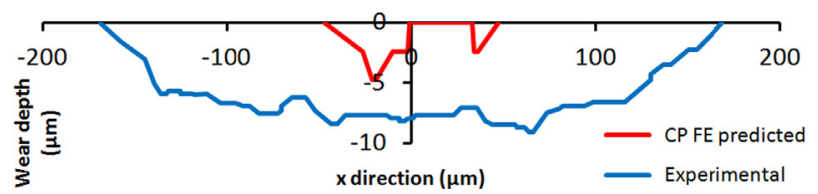

Fig. 24. Comparison of CPFE (SET 1) predicted and sample measured wear profile after 56,669 cycles at $\sigma_{a m p}=225 \mathrm{MPa}$. 
existing prediction methods for wear scar shape in gross slip can only predict a U-shape. Mohd Tobi et al. [53] presented a detailed analysis of the interaction between $J_{2}$ plasticity and wear. It was shown that the characteristics of predicted plastic strain and SWT damage accumulation are significantly different for the gross sliding and partial slip cases and that the differences can be correlated with the observed differences in surface morphology and surface cracking. It was also shown that the location of plasticity due to shear yielding at the surface promotes wear across the contact region for gross sliding and at the stick-slip interface for partial slip. It was furthermore observed that the predicted accumulated effective plastic strain evolutions produced W-shaped trends which corresponding to the measured wear profiles. The $\mathrm{W}$-shape plastic strain distributions were induced by the simulation of wear-induced material removal and were interpreted to correspond to the 'ploughing' effect. Similar observations can be made with regards Figs. 14(a) and 15(a) where distinct peaks in accumulated plastic slip produce comparable distributions to those in [53]. The importance of plasticity effects within fretting is highlighted by the fact that the Archard based wear simulation presented in [53], which is dependent on pressure and slip, always produces U-shaped wear scars and is not capable of capturing the localised plasticity wear effects observed experimentally. It is argued in this work that wear is a micro-cracking process and the accumulated plastic slip distributions are analogous to fretting wear and delamination observed in Mohd Tobi et al. [53] and in the current experimental work [18]. The presence of unfavourably orientated grains within the wear scar region may also explain W-shaped scars. These "harder" grains have not experienced the same amount of accumulated plastic slip as a result of slip system orientation.

(2) The hypothesis that CP can be used to predict, and hence simulate, wear, appears to have greater validity for very early numbers of cycles, where plasticity phenomena of surface asperities (e.g. see Mulvihill et al. [56]) can be expected to dominate wear and friction behaviour. However, it is difficult to measure wear for small numbers of cycles, due to competing length-scales of surface roughness and early wear damage; similarly, in the present work, it has only been possible to compare the CPFE wear prediction to a measured wear scar after a large number of cycles. It follows that CPFE wear predictions may show a greater degree of success if extended to explicitly simulate material removal and with inclusion of surface roughness geometry, i.e. using $p_{c y c}$ as a material removal (wear) parameter in an approach otherwise similar to that of Ding et al. [57]. The predicted location of cracking is $-0.659 a_{0}$ for $\sigma_{a m p}=225 \mathrm{MPa}$ for example in Fig. 15(a), where as the experimentally observed cracks occur at the contact edges $\approx-10 a_{0}$ due to the significantly greater increase in wear scar and hence contact width in the experiments. The predicted wear scar is significantly smaller than the measured experimental wear scar (see Fig. 24) since material removal [10] is not simulated in this work. Therefore, the specific location of predicted cracking is different from what is observed experimentally, although on the correct side of the contact.

(3) Multiple experimental wear profiles taken at different transverse locations through the fretting specimen show both U-shaped and W-shaped 2D wear scars. The measured W-shaped wear scars have not been shown here, but are shown in [42]. It is therefore possible to assume that if a three dimensional $\mathrm{CP}$ model was implemented here, similar wear profile distributions would be evident at different transverse locations throughout the thickness of the FE substrate. The microstructure-sensitive damage approach clearly demonstrates the ability to predict W-shaped wear scars and would be further enhanced by measurement of the microstructure with respect to grain orientation. This gives further evidence to suggest that the present microstructuresensitive model has a superior ability to realistically capture the experimental behaviour of $\mathrm{FF}$, as compared to macro-scale plasticity models.

\section{Conclusion}

The key conclusion is that grain orientation is shown to have a significant effect on the fretting fatigue performance of a laboratory arrangement, as compared to plain fatigue performance. In design for gross-slip, particularly with a view to quantification of micro-structure sensitive scatter, it is critical to consider the ratios of contact width and slip amplitude to grain size. For reliable prediction of number of cycles to fretting fatigue crack nucleation and hence life, a micro-structure sensitive framework is required. This is particularly so when the latter ratios are small, i.e. large grain size relative to contact and non-uniform slip region sizes. It was shown that consideration of random orientation in such a microstructure-sensitive framework facilitated interpretation and representation of the complex interaction effects of relative slip (fretting, surface damage) and fatigue (sub-surface) damage with respect to stress amplitude effect on fretting fatigue (nucleation) life. Furthermore, the proposed microstructure-sensitive approach was shown to have the ability to predict, from a fundamental, plasticity basis, reasonably realistic wear scars, including, in particular, a W-shaped scar, which is consistent with commonlyobserved shapes, and not achievable by macro-scale plasticity.

\section{Acknowledgements}

The authors would like to acknowledge the assistance of Ms Caoimhe Sweeney in the generation of the Voronoi Tesselation mesh for this paper.

\section{References}

[1] Wavish PM, Houghton D, Ding J, Leen SB, Williams EJ, McColl IR. A multiaxial fretting fatigue test for spline coupling contact. Fatigue Fract Eng Mater Struct 2009;32(4):325-45.

[2] Zhang T, Harrison NM, McDonnell PF, McHugh PE, Leen SB. A finite element methodology for wear-fatigue analysis for modular hip implants. Tribol Int 2013;65:113-27.

[3] Vidner J, Leidich E. Enhanced Ruiz criterion for the evaluation of crack initiation in contact subjected to fretting fatigue. Int J Fatigue 2007;29(9-11):2040-9.

[4] Benhamena A, Amrouche A, Talha A, Benseddiq N. Effect of contact forces on fretting fatigue behavior of bolted plates: numerical and experimental analysis. Tribol Int 2012:48:237-45.

[5] Araújo JA, Nowell D. The effect of rapidly varying contact stress fields on fretting fatigue. Int J Fatigue 2002;24(7):763-75.

[6] Sum WS, Williams EJ, Leen SB. Finite element, critical-plane, fatigue life prediction of simple and complex contact configurations. Int J Fatigue 2005;27 (4):403-16.

7] Dobromirski JM. Variables in the fretting process: are there 50 of them? Standardisation of fretting fatigue test methods and equipment. ASTM. Waterhouse RB, Attia MH. (editors); 1992.

[8] Vingsbo O, Soderberg D. On fretting maps. Wear 1988;126:131-47.

[9] Jin O, Mall S. Effects of slip on fretting behavior: experiments and analyses. Wear 2004;256(7-8):671-84

[10] Madge JJ, Leen SB, McColl IR, Shipway PH. Contact-evolution based prediction of fretting fatigue life: effect of slip amplitude. Wear 2007:262(9-10):1159-70.

[11] Ding J, Bandak G, Leen SB, Williams EJ, Shipway PH. Experimental characterisation and numerical simulation of contact evolution effect on fretting crack nucleation for Ti-6Al-4V. Tribol Int 2009;42(11-12):1651-62.

[12] Ambrico JM, Begley MR. Plasticity in fretting contact. J Mech Phys Solids 2000;48(11):2391-417.

[13] Goh C-H, McDowell DL, Neu RW. Characteristics of plastic deformation field in polycrystalline fretting contacts. Int J Fatigue 2003;25(9-11):1047-58.

[14] Mayeur JR, McDowell DL, Neu RW. Crystal plasticity simulations of fretting of Ti-6Al-4V in partial slip regime considering effects of texture. Comput Mater Sci 2008;41(3):356-65.

[15] Zhang M, Neu RW, McDowell DL. Microstructure-sensitive modeling: application to fretting contacts. Int J Fatigue 2009;31(8-9):1397-406. 
[16] Dick T, Cailletaud G. Fretting modelling with a crystal plasticity model of Ti6Al4V. Comput Mater Sci 2006;38(1):113-25.

[17] McCarthy OJ, McGarry JP, Leen SB. Micro-mechanical modelling of fretting fatigue crack initiation and wear in Ti-6Al-4V. International Journal of Fatigue 2013, http://dx.doi.org/10.1016/j.ijfatigue.2013.04.019.

[18] McCarthy OJ, McGarry JP, Leen SB. Microstructure-sensitive prediction and experimental validation of fretting fatigue. Wear 2013;305:100-14.

[19] Ratsimba CHH, McColl IR, Williams EJ, Leen SB, Soh HP. Measurement, analysis and prediction of fretting wear damage in a representative aeroengine spline coupling. Wear 2004;257(11):1193-206.

[20] Lemaitre J, Chaboche J-L. Mechanics of solid materials. Chichester: Cambridge University Press; 1994.

[21] Hyde CJ, Sun W, Leen SB. Cyclic thermo-mechanical material modelling and testing of 316 stainless steel. Int J Pressure Vessels Piping 2010;87(6):365-72.

[22] Khan AS, Huang S. Continuum theory of plasticity. Cambridge: John Wiley \& Sons; 1995

[23] McCarthy OJ, McGarry JP, Leen SB. A finite element study of microstructuresensitive plasticity and crack nucleation in fretting. Comput Mater Sci 2011;50 (8):2439-58

[24] Huang Y. A user-material subroutine incorporating single crystal plasticity in the ABAQUS finite element program. Harvard University Report, vol. Mech $178 ; 1991$.

[25] Manonukul A, Dunne FPE. High and low cycle fatigue crack initiation using polycrystal plasticity. Proc R Soc 2004;460:1881-903.

[26] Sweeney CA, McHugh PE, McGarry JP, Leen SB. Micromechanical methodology for fatigue in cardiovascular stents. Int J Fatigue 2012;44:202-16.

[27] Sweeney CA, Vorster W, Leen SB, Sakurada E, McHugh PE, Dunne FPE. The role of elastic anisotropy, length scale and crystallographic slip in fatigue crack nucleation. J Mech Phys Solids 2013;61(5):1224-40.

[28] Smith KN, Watson P, Topper TH. A stress-strain function for the fatigue of metals. J Mater 1970;15:767-78.

[29] Socie D. Multiaxial fatigue damage models. J Eng Mater Technol 1987;109 (4):293-8.

[30] Fatemi A, Socie D. Multiaxial fretting fatigue testing and prediction for splined couplings. Fatigue Fract Eng Mater Struct 1998;11(3):149-65.

[31] Leen SB, Hyde TH, Ratsimba CHH, Williams EJ, McColl IR. An investigation of the fatigue and fretting performance of a representative aero-engine spline coupling. I Strain Anal Eng Des 2002;37(6):565-83.

[32] Houghton D, Wavish PM, Williams EJ, Leen SB. Multiaxial fretting fatigue testing and prediction for splined couplings. Int J Fatigue 2009;31(11-12) 1805-15.

[33] Szolwinski MP, Farris TN. Observation, analysis and prediction of fretting fatigue in 2024-T351 aluminum alloy. Wear 1998;221(1):24-36.

[34] Madge IJ, Leen SB, Shipway PH. A combined wear and crack nucleationpropagation methodology for fretting fatigue prediction. Int J Fatigue 2008;30 (9):1509-28.

[35] Bernardo AT, Araújo JA, Mamiya EN. Proposition of a finite element-based approach to compute the size effect in fretting fatigue. Tribol Int 2006;39 (10):1123-30.
[36] Suresh S. Fatigue of materials. Cambridge: University Press; 2001.

[37] El Haddad MH, Topper TH, Smith KN. Prediction of non propagating cracks. Eng Fract Mech 1979;11(3):573-84.

[38] Obrtlik K, Polák J, Hájek M, Vašek A. Short fatigue crack behaviour in 316L stainless steel. Int J Fatigue 1997;19(6):471-5.

[39] Nicholas T, Hutson A, John R, Olson S. A fracture mechanics methodology assessment for fretting fatigue. Int J Fatigue 2003;25(9-11):1069-77.

[40] Fett T, Munz D. Stress intensity factors and weight functions. Southampton: Computational Mechanics Publications; 1997.

[41] Walker J. The effect of stress ratio during crack propagation and fatigue for 2024-T3 and 7075-T6 aluminum. Effects of environment and complex load history on fatigue life. ASTM STP 462, p. 1-14; 1970.

[42] McCarthy OJ. A study of microstructure-sensitive crack nucleation and wear in fretting. PhD Thesis, NUI Galway; 2013.

[43] McColl IR, Ding J, Leen SB. Finite element simulation and experimenta validation of fretting wear. Wear 2004;256(11-12):1114-27.

[44] Harewood FJ, McHugh PE. Investigation of finite element mesh independence in rate dependent materials. Comput Mater Sci 2006;37(4):442-53.

[45] Ding J, Houghton D, Williams EJ, Leen SB. Simple parameters to predict effect of surface damage on fretting fatigue. Int J Fatigue 2011:33(3):332-42.

[46] Liu Y, Mahadevan S. Threshold stress intensity factor and crack growth rate prediction under mixed-mode loading. Eng Fract Mech 2007;74(3):332-45.

[47] McGarry JP, O'Donnell BP, McHugh PE, O'Cearbhaill E, McMeeking RM. Computational examination of the effect of material inhomogeneity on the necking of stent struts under tensile loading. J Appl Mech 2007;74:978-90.

[48] Feerick EM, (Cheryl) Liu X, McGarry P. Anisotropic mode-dependent damage of cortical bone using the extended finite element method (XFEM). J Mech Behav Biomed Mater 2013;20:77-89.

[49] You X, Connolley T, McHugh PE, Cuddy H, Motz C. A combined experimental and computational study of deformation in grains of biomedical grade 316LVM stainless steel. Acta Mater 2006;54(18):4825-40.

[50] Fouvry S, Duó P, Perruchaut P. A quantitative approach of Ti-6Al-4V fretting damage: friction, wear and crack nucleation. Wear 2004;257(9-10):916-29.

[51] Ding J, McColl IR, Leen SB, Shipway PH. A finite element based approach to simulating the effects of debris on fretting wear. Wear 2007;263(1-6):481-91.

[52] Hutchings IM. Tribology: friction and wear of engineering materials. Oxford: Butterworth-Heinemann; 1992.

[53] Mohd Tobi AL, Ding J, Bandak G, Leen SB, Shipway PH. A study on the interaction between fretting wear and cyclic plasticity for Ti-6Al-4V. Wear 2009;267(1-4):270-82.

[54] Magaziner RS, Jain VK, Mall S. Wear characterization of Ti-6Al-4V under fretting-reciprocating sliding conditions. Wear 2008;264(11-12):1002-14.

[55] Fouvry S, Kapsa P, Zahouani H, Vincent L. Wear analysis in fretting of hard coatings through a dissipated energy concept. Wear 1997;203-204:393-403.

[56] Mulvihill DM, Kartal ME, Nowell D, Hills DA. An elastic-plastic asperity interaction model for sliding friction. Tribol Int 2011;44(12):1679-94.

[57] Ding J, Leen SB, Williams EJ, Shipway PH. A multi-scale model for fretting wear with oxidation-debris effects. Proc Inst Mech Eng, Part J: J Eng Tribol 2009;223 (7):1019-31. 